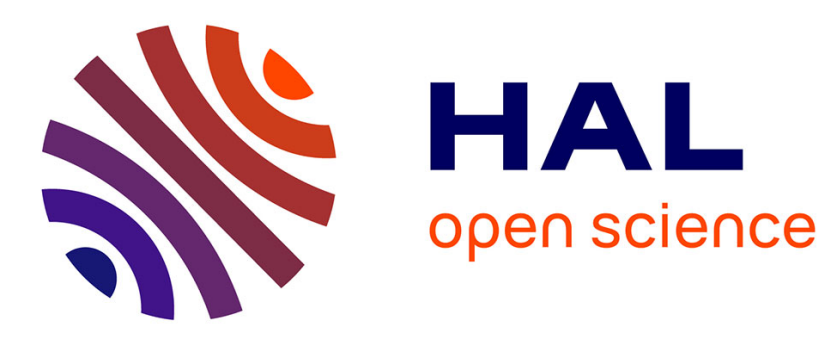

\title{
CTL Model Checking of Self Modifying Code
}

\author{
Tayssir Touili, Xin Ye
}

\section{To cite this version:}

Tayssir Touili, Xin Ye. CTL Model Checking of Self Modifying Code. Proceedings of the 25th INTERNATIONAL CONFERENCE ON ENGINEERING OF COMPLEX COMPUTER SYSTEMS (ICECCS 2020), 2020. hal-03034019

\section{HAL Id: hal-03034019 \\ https://cnrs.hal.science/hal-03034019}

Submitted on 1 Dec 2020

HAL is a multi-disciplinary open access archive for the deposit and dissemination of scientific research documents, whether they are published or not. The documents may come from teaching and research institutions in France or abroad, or from public or private research centers.
L'archive ouverte pluridisciplinaire HAL, est destinée au dépôt et à la diffusion de documents scientifiques de niveau recherche, publiés ou non, émanant des établissements d'enseignement et de recherche français ou étrangers, des laboratoires publics ou privés. 


\section{CTL Model Checking of Self Modifying Code}

\author{
Tayssir Touili \\ LIPN, CNRS and University Paris 13
}

\author{
$\mathrm{Xin} \mathrm{Ye}$ \\ Shanghai Key Lab. of Trustworthy Comput., ECNU and LIPN
}

\begin{abstract}
Self-modifying code is extensively used to obfuscate malware and to make reverse engineering harder. It consists in code that can modify its own instructions during the execution. Being able to analyse such code is crucial nowadays. In this paper, we consider the CTL model-checking problem of self modifying code. To model such programs, we use Self Modifying Pushdown Systems (SM-PDS), an extension of pushdown systems whose set of rules can be modified during execution. We reduce the CTL model-checking problem to the emptiness problem of Self-Modifying Alternating Büchi pushdown systems (SMABPDS). We implemented our techniques in a tool. We obtained encouraging results. In particular, our tool was able to detect several self-modifying malwares; it could even detect several malwares that well-known antiviruses such as McAfee, Norman, BitDefender, Kinsoft, Avira, eScan, Kaspersky, Qihoo-360, Avast, and Symantec failed to detect.
\end{abstract}

\section{INTRODUCTION}

Self-modifying code is code that modifies its own instructions during its execution. It is usually used to enhance the difficulty of disassembling in reverse engineering. It is not only applied for software protection but also to make malwares harder to analyze and detect by static analysis approaches. Therefore, it is vital to analyze the programs equipped with self-modifying codes. In [प], [2], [3] , [4], [5], [6], []], [], self-modifying codes implemented by packing and unpacking techniques have been well studied. Analysis tools for such codes are available [9], [10].

In this work, we consider another kind of implementation of self-modifying codes: self-modifying instructions. These are instructions that can read and write to memory, like the mov instruction that is able to copy data from one memory location to another. Using such self-modifying instructions can make malware detection harder. Fig. 凹 shows how malware can use self-modifying instructions to evade from static analysis techniques. This figure shows a fragment of the malware Bagle.J equipped with such self-modifying instructions. First let us recall the semantics of the mov instruction. It copies the data item referred to by its second operand (register or memory location) into its first operand. In Fig. M, in the box on the left, we give, respectively, the binary code, the addresses of the different instructions, and the corresponding assembly code, obtained by translating syntactically the binary code at each address. For example, $\mathrm{f} f$ is the binary code of the instruction push. Thus, the first line is translated to push $0 \mathrm{~b}$. The second instruction mov $0 \times 20 \times c$ will replace the first byte at address $0 \times 2$ by $0 \times c$. Thus, at address $0 \times 2$, ff $\mathrm{Ob}$ is replaced by $\mathrm{OCOb}$, i.e., the instruction push $\mathrm{Ob}$ is replaced by jmp $0 \mathrm{~b}$. If we analyse this code without taking into account the fact that mov $0 \times 2 \quad 0 \times c$ is a self-modifying instruction, then, we will obtain the Control Flow Graph "CFG a", and we will reach the conclusion that the Bagle malicious behaviour implemented at address $0 \mathrm{~b}$ by the API functions RegCreateKeyA, RegDeleteValueA, and RegCloseKey is not reachable. However, the actual CFG is "CFG b", where the malicious fragment of the malware Bagle.J that starts at address $\mathrm{Ob}$ is reached and will be executed.

It can be seen from this example that self-modifying codes can make malware detection harder, and that the mov instruction is able to modify instructions of the program successfully via its ability to read and write the memory. Thus, it is crucial to be able to analyse this kind of self-modifying code.

Self Modifying Pushdown Systems (SM-PDS) was proposed in [1]] as a perfect model to represent such selfmodifying codes. Indeed, a SM-PDS is an extension of standard Pushdown Systems (PDS) with self-modifying transition rules that modify the set of the rules of the PDS during the execution. This allows to represent the self-modifying instructions of the program. Moreover, SM-PDSs allow to keep track of the program's stack, which is very important for malware detection. Indeed, as decribed in [12], many obfuscation techniques rely on operations over the stack. Therefore, it is important for malware detection to have analysis techniques that can deal with the program stack.

[1]] proposed efficient reachability analysis techniques for SM-PDSs. However, reachability is not enough to describe several malicious behaviors. For example, [13] showed that the branching-time temporal logic CTL is needed to represent several malicious behaviors. In this work, we go one step further and consider the CTL model-checking problem for SM-PDSs. This allows to detect CTL-like malicious behaviors on self-modifying code. We reduce this problem to the emptiness checking of Self-modifying Alternating Büchi Pushdown Systems (SM-ABPDS), and we propose an algorithm that computes a finite automaton that characterizes the set of configurations accepted by the SM-ABPDS. We implemented our techniques in a tool for self-modifying code analysis. We obtained encouraging results. Indeed, our tool was able to detect more than 700 self-modifying malwares. Amongst these malwares, several could not be detected by well known and widely used commercial anti-viruses such as McAfee, Norman, BitDefender, Kinsoft, Avira, eScan, Kaspersky, Qihoo360, Avast, and Symantec.

Outline. The rest of the paper is organized as follows: In section 2, we recall the definition of SM-PDS. Section 3 reviews 

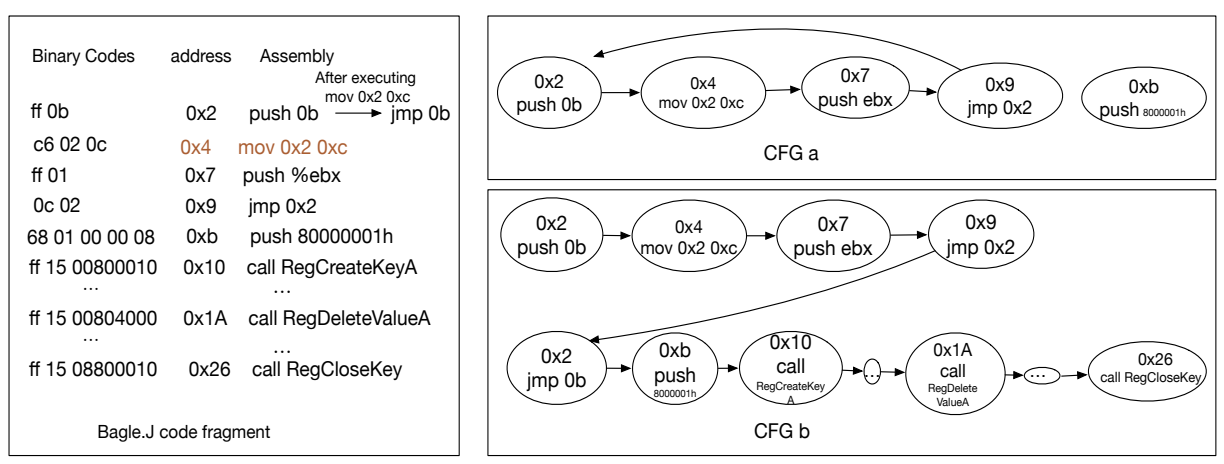

Fig. 1: An Example of Self-modifying Code

the definition of CTL and shows how CTL model checking on SM-PDSs can be reduced to the emptiness analysis on SMABPDSs. In Section 4, we give our algorithm that computes a finite automaton representing the set of configurations accepted by a SM-ABPDS. Our experiments are described in Sections 5. For lack of space, the proofs are ommitted. They can be found in the full version [14]. Moreover, the full version [114] contains also bigger experimental tables.

Related Work. Model checking and static analysis techniques were extensively applied for the analysis of binary code [I], [2], [3], [5], [15]. Malicious behaviors were described by temporal Logics in [16], [5], [15], [177], [118]. However, these works cannot deal with self-modifying code.

Formal semantics of self-modifying code was proposed in [19], [20], [21]. However, these specifications are too abstract to be used in practice. A new representation of selfmodifying code called State Enhanced-Control Flow Graph (SE-CFG) was proposed in [22]. SE-CFG extends standard control flow graphs with a new data structure, keeping track of the possible states programs can reach, and with edges that can be conditional on the state of the target memory location. Unlike SM-PDSs, this representation does not allow to take into account the stack of the program. Abstract interpretation techniques that compute an over-approximation of the set of reachable states of a self-modifying program were proposed in [23]. Combining static and dynamic analysis techniques to analyse self-modifying programs was applied in [24]. These techniques [23], [24] cannot handle the program's stack. Unpacking binary code is considered in [25], [26], [27], [22]]. These works do not consider self-modifying mov instructions.

CTL model-checking for pushdown systems (PDS) was considered in [28], [133]. In [13], this problem is reduced to the emptiness problem in Alternating Büchi Pushdown Systems. In this work, we extend this approach to SM-PDSs. CTL model-checking for SM-PDSs can be reduced to CTL modelchecking for PDSs. However, as witnessed by the results in the experiments section, this reduction is not efficient. We propose in this paper a direct and more efficient approach.

\section{Self Modifying Pushdown Systems}

In this section, we recall the definition of Self-modifying Pushdown Systems [II]].

Definition 1. A Self-modifying Pushdown System (SM-PDS) is a tuple $\mathcal{P}=\left(P, \Gamma, \Delta, \Delta_{c}\right)$, where $P$ is a finite set of control points, $\Gamma$ is a finite set of stack symbols, $\Delta \subseteq(P \times \Gamma) \times(P \times$ $\left.\Gamma^{*}\right)$ is a finite set of transition rules, and $\Delta_{c} \subseteq P \times\left(\Delta \cup \Delta_{c}\right) \times$ $\left(\Delta \cup \Delta_{c}\right) \times P$ is a finite set of modifying transition rules. If $\left((p, \gamma),\left(p^{\prime}, w\right)\right) \in \Delta$, we also write $\langle p, \gamma\rangle \hookrightarrow\left\langle p^{\prime}, w\right\rangle \in \Delta$. If $\left(p, r_{1}, r_{2}, p^{\prime}\right) \in \Delta_{c}$, we also write $p \stackrel{\left(r_{1}, r_{2}\right)}{\longrightarrow} p^{\prime} \in \Delta_{c}$. A Pushdown System (PDS) is a SM-PDS where $\Delta_{c}=\emptyset$.

Intuitively, a Self-modifying Pushdown System is a Pushdown System that can dynamically modify its set of rules during the execution time: rules $\Delta$ are standard PDS transition rules, while rules $\Delta_{c}$ modify the current set of transition rules: $\langle p, \gamma\rangle \hookrightarrow\left\langle p^{\prime}, w\right\rangle \in \Delta$ expresses that if the SM-PDS is in control point $p$ and has $\gamma$ on top of its stack, then it can move to control point $p^{\prime}$, pop $\gamma$ and push $w$ onto the stack, while $p \stackrel{\left(r_{1}, r_{2}\right)}{\longrightarrow} p^{\prime} \in \Delta_{c}$ expresses that when the PDS is in control point $p$, then it can move to control point $p^{\prime}$, remove the rule $r_{1}$ from its current set of transition rules, and add the rule $r_{2}$.

Formally, a configuration of a SM-PDS is a tuple $c=$ $(\langle p, w\rangle, \theta)$ where $p \in P, w \in \Gamma^{*}$ and $\theta \subseteq \Delta \cup \Delta_{c}$ is the current set of transition rules of the SM-PDS. $\theta$ is called the current phase of the SM-PDS. A SM-PDS defines a transition relation $\Rightarrow_{\mathcal{P}}$ between configurations as follows: Let $p \in P, \gamma \in \Gamma, w \in \Gamma^{*}$, and $\theta \subseteq \Delta \cup \Delta_{c}$, then:

1) if $r \in \Delta_{c}$ is of the form $r=p \stackrel{\left(r_{1}, r_{2}\right)}{\longrightarrow} p^{\prime}$, such that $r_{1} \in \theta$, then $(\langle p, w\rangle, \theta) \Rightarrow_{\mathcal{P}}\left(\left\langle p^{\prime}, w\right\rangle, \theta^{\prime}\right)$, where $\theta^{\prime}=$ $\left(\theta \backslash\left\{r_{1}\right\}\right) \cup\left\{r_{2}\right\}$. In other words, the transition rule $r$ updates the current set of transition rules $\theta$ by removing $r_{1}$ from it and adding $r_{2}$ to it.

2) if $r \in \Delta$ is of the form $r=\langle p, \gamma\rangle \hookrightarrow\left\langle p^{\prime}, w^{\prime}\right\rangle \in \Delta$, then $(\langle p, \gamma w\rangle, \theta) \Rightarrow_{\mathcal{P}}\left(\left\langle p^{\prime}, w^{\prime} w\right\rangle, \theta\right)$ : the transition rule $r$ moves the control point from $p$ to $p^{\prime}$, pops $\gamma$ from the stack and pushes $w^{\prime}$ onto the stack. This transition keeps the current set of transition rules $\theta$ unchanged.

Let $\Rightarrow{ }_{\mathcal{P}}^{*}$ be the transitive, reflexive closure of $\Rightarrow_{\mathcal{P}}$. A path of $\mathcal{P}$ is a sequence of configurations $c_{0} c_{1} \ldots$ s.t. for every $i \geq 0$, 
$c_{i} \Rightarrow_{\mathcal{P}} c_{i+1}$. Then, for every $i \geq 0, c_{i+1}$ is an immediate successor of $c_{i}$, and $c_{i}$ is an immediate predecessor of $c_{i+1}$.

To simplify the presentation, we assume w.l.o.g. that $P=$ $P_{N} \cup P_{c}$ s.t. $P_{N} \cap P_{c}=\emptyset$, and for every $\langle p, \gamma\rangle \hookrightarrow\left\langle p^{\prime}, w\right\rangle \in \Delta$, $p \in P_{N}$ and for every $p \stackrel{\left(r_{1}, r_{2}\right)}{\longrightarrow} p^{\prime} \in \Delta_{c}, p \in P_{c}$ (we can always transform the rules of a given SM-PDS into equivalent ones that meet this condition).

SM-PDS vs. PDS. Let $\mathcal{P}=\left(P, \Gamma, \Delta, \Delta_{c}\right)$ be a SM-PDS. It was shown in [11] that $\mathcal{P}$ can be translated to an equivalent pushdown system. The basic idea is to encode phases in the control points of the PDS (since the number of phases is finite). However, this translation is not efficient since the number of control points of the equivalent PDS is $|P| \cdot 2^{\mathcal{O}\left(|\Delta|+\left|\Delta_{c}\right|\right)}$.

From Self-modifying Codes to SM-PDS. SM-PDSs can be used to model self-modifying binary code as described in [11]. Our translation relies on the tool Jakstab [29] to disassemble binary code, determine indirect jumps, determine the program's instructions, and compute information about the values of the registers and the memory locations at each control point of the program. After getting the assembly program corresponding to the binary code using Jakstab [29], we translate it into a SM-PDS as described in [11]. In our translation, the control locations store the control points of the binary program and the stack mimics the program's stack. The non self-modifying instructions of the program define the rules $\Delta$ of the SM-PDS (which are standard PDS rules), and can be obtained following the translation of [15] that models non self-modifying instructions of the program by a PDS. As for the self-modifying instructions of the program, they define the set of changing rules $\Delta_{c}$. For more details, we refer the reader to [II]].

\section{CTL MODEL-CHECKING ON SM-PDSS}

\section{A. The Computation Tree Logic CTL}

Let $A t$ be a finite set of atomic propositions. CTL formulas over $A t$ are defined as follows (where $A \in A t$ ):

$$
\begin{array}{r}
\varphi:=A|\neg A| \varphi \vee \varphi|\varphi \wedge \varphi| A X \varphi|E X \varphi| \\
A[\varphi U \varphi]|E[\varphi U \varphi]| A[\varphi \widetilde{U} \varphi] \mid E[\varphi \widetilde{U} \varphi] .
\end{array}
$$

Given a CTL formula $\varphi$, the closure $\operatorname{cl}(\varphi)$ is the set of all the subformulae of $\varphi$, including $\varphi$. Let $\mathcal{P}=\left(P, \Gamma, \Delta, \Delta_{c}\right)$ be a SM-PDS, $\nu: P \rightarrow 2^{A t}$ be a labelling function mapping to each control location $p \in P$ a set of atomic propositions. The satisfiability relation of a CTL formula $\varphi$ at a configuration $\left(\left\langle p_{0}, w_{0}\right\rangle, \theta_{0}\right)$ (denoted by $\left.\left(\left\langle p_{0}, w_{0}\right\rangle, \theta_{0}\right) \models_{\nu} \varphi\right)$ is defined as follows:

- $\left(\left\langle p_{0}, w_{0}\right\rangle, \theta_{0}\right) \models{ }_{\nu} A$ iff $A \in \nu\left(p_{0}\right)$,

- $\left(\left\langle p_{0}, w_{0}\right\rangle, \theta_{0}\right) \models{ }_{\nu} \neg A$ iff $A \notin \nu\left(p_{0}\right)$,

- $\left(\left\langle p_{0}, w_{0}\right\rangle, \theta_{0}\right) \quad \models_{\nu} \quad \varphi_{1} \vee \varphi_{2}$ iff $\left(\left\langle p_{0}, w_{0}\right\rangle, \theta_{0}\right) \quad \models_{\nu} \quad \varphi_{1} \quad$ or $\left(\left\langle p_{0}, w_{0}\right\rangle, \theta_{0}\right) \models{ }_{\nu} \varphi_{2}$,

- $\left(\left\langle p_{0}, w_{0}\right\rangle, \theta_{0}\right) \quad \models{ }_{\nu} \quad \varphi_{1} \wedge \varphi_{2}$ iff $\left(\left\langle p_{0}, w_{0}\right\rangle, \theta_{0}\right) \quad \models_{\nu} \quad \varphi_{1} \quad$ and $\left(\left\langle p_{0}, w_{0}\right\rangle, \theta_{0}\right) \models_{\nu} \varphi_{2}$,

- $\left(\left\langle p_{0}, w_{0}\right\rangle, \theta_{0}\right) \models_{\nu} \quad A X \varphi$ iff $\left(\left\langle p_{1}, w_{1}\right\rangle, \theta_{1}\right) \models_{\nu} \quad \varphi$ for every successor $\left(\left\langle p_{1}, w_{1}\right\rangle, \theta_{1}\right)$ of $\left(\left\langle p_{0}, w_{0}\right\rangle, \theta_{0}\right)$,

- $\left(\left\langle p_{0}, w_{0}\right\rangle, \theta_{0}\right) \models_{\nu} \quad E X \varphi$ iff $\left(\left\langle p_{1}, w_{1}\right\rangle, \theta_{1}\right) \models_{\nu} \varphi$ for some successor $\left(\left\langle p_{1}, w_{1}\right\rangle, \theta_{1}\right)$ of $\left(\left\langle p_{0}, w_{0}\right\rangle, \theta_{0}\right)$,
- $\left(\left\langle p_{0}, w_{0}\right\rangle, \theta_{0}\right) \models_{\nu} A\left[\varphi_{1} U \varphi_{2}\right]$ iff for every path

$$
\left(\left\langle p_{0}, w_{0}\right\rangle, \theta_{0}\right)\left(\left\langle p_{1}, w_{1}\right\rangle, \theta_{1}\right) \cdots
$$

of $\mathcal{P}$ starting from $\left(\left\langle p_{0}, w_{0}\right\rangle, \theta_{0}\right), \exists i \geq 0$ s.t. $\left(\left\langle p_{i}, w_{i}\right\rangle, \theta_{i}\right) \models{ }_{\nu} \varphi_{2}$ and $\forall 0 \leq j<i,\left(\left\langle p_{j}, w_{j}\right\rangle, \theta_{j}\right) \mid{ }_{\nu} \varphi_{1}$.

- $\left(\left\langle p_{0}, w_{0}\right\rangle, \theta_{0}\right) \models{ }_{\nu} E\left[\varphi_{1} U \varphi_{2}\right]$ iff there exists a path

$$
\left(\left\langle p_{0}, w_{0}\right\rangle, \theta_{0}\right)\left(\left\langle p_{1}, w_{1}\right\rangle, \theta_{1}\right) \cdots
$$

of $\mathcal{P}$ starting from $\left(\left\langle p_{0}, w_{0}\right\rangle, \theta_{0}\right), \exists i \geq 0$ s.t. $\left(\left\langle p_{i}, w_{i}\right\rangle, \theta_{i}\right) \models{ }_{\nu} \varphi_{2}$, $\forall 0 \leq j<i,\left(\left\langle p_{j}, w_{j}\right\rangle, \theta_{j}\right) \models{ }_{\nu} \varphi_{1}$.

- $\left(\left\langle p_{0}, w_{0}\right\rangle, \theta_{0}\right) \models_{\nu} A\left[\varphi_{1} \widetilde{U} \varphi_{2}\right]$ iff for every path

$$
\left(\left\langle p_{0}, w_{0}\right\rangle, \theta_{0}\right)\left(\left\langle p_{1}, w_{1}\right\rangle, \theta_{1}\right) \cdots
$$

of $\mathcal{P}$ starting from $\left(\left\langle p_{0}, w_{0}\right\rangle, \theta_{0}\right), \forall i \geq 0$, if $\left(\left\langle p_{i}, w_{i}\right\rangle, \theta_{i}\right) \not \not_{\nu} \varphi_{2}$, then $\exists 0 \leq j<i,\left(\left\langle p_{j}, w_{j}\right\rangle, \theta_{j}\right) \models{ }_{\nu} \varphi_{1}$.

- $\quad\left(\left\langle p_{0}, w_{0}\right\rangle, \theta_{0}\right) \stackrel{\models}{\nu} \quad E\left[\varphi_{1} \widetilde{U} \varphi_{2}\right]$ iff $\exists \quad \exists \quad$ a path $\left(\left\langle p_{0}, w_{0}\right\rangle, \theta_{0}\right)\left(\left\langle p_{1}, w_{1}\right\rangle, \theta_{1}\right) \cdots \quad$ of $\quad \mathcal{P} \quad$ starting with $\left(\left\langle p_{0}, w_{0}\right\rangle, \theta_{0}\right)$, s.t. $\forall i \geq 0$, if $\left(\left\langle p_{i}, w_{i}\right\rangle, \theta_{i}\right) \quad \not \nvdash_{\nu} \quad \varphi_{2}$, then $\exists 0 \leq j<i,\left(\left\langle p_{j}, w_{j}\right\rangle, \theta_{j}\right) \models_{\nu} \varphi_{1}$.

Standard CTL operators can be expressed by the above operators: $\mathbf{E F} \psi=\mathbf{E}[\operatorname{true} \mathbf{U} \psi], \mathbf{A F} \psi=\mathbf{A}[\operatorname{true} \mathbf{U} \psi], \mathbf{E G} \psi=$ $\mathbf{E}[$ false $\widetilde{U} \psi], \mathbf{A G} \psi=\mathbf{A}[$ false $\widetilde{U} \psi]$.

\section{B. Self-modifying Alternating Büchi Pushdown Systems}

Definition 2. A Self Modifying Alternating Büchi Pushdown System (SM-ABPDS) is a tuple $\mathcal{B P}=\left(P, \Gamma, \Delta, \Delta_{c}, F\right)$, where $P$ is a finite set of control points, $\Gamma$ is a finite set of stack symbols, $F$ is the set of final states, $\Delta \subseteq$ $(P \times \Gamma) \times 2^{2^{\Delta \cup \Delta_{c}} \cup\{-\}} \times 2^{P \times \Gamma^{*}} \quad$ is a finite set of transition rules in the form $\langle p, \gamma\rangle \stackrel{\left[\sigma_{1}, \cdots, \sigma_{n}\right]}{\longrightarrow}\left\{\left\langle p_{1}, w_{1}\right\rangle, \cdots,\left\langle p_{n}, w_{n}\right\rangle\right\}$ where $\left[\sigma_{1}, \cdots, \sigma_{n}\right]$ is an ordered set and $\forall 1 \leq i \leq n, \sigma_{i}$ is either a set of rules $\sigma_{i} \subseteq \Delta \cup \Delta_{c}$ or $\sigma_{i}=-$, and $\Delta_{c} \subseteq P \times 2^{\Delta \cup \Delta_{c}} \times 2^{\Delta \cup \Delta_{c}} \times P$ is a finite set of modifying transition rules in the form $p \stackrel{\left(\sigma, \sigma^{\prime}\right)}{\longrightarrow} p^{\prime}$ where $\sigma, \sigma^{\prime} \subseteq \Delta \cup \Delta_{c}$. A configuration of a SM-ABPDS is a tuple of the form $(\langle p, w\rangle, \theta)$ where $p \in P, w \in \Gamma^{*}$ and $\theta \subseteq \Delta \cup \Delta_{c}$ is the current phase.

$\mathcal{B P}$ defines the transition relation $\Rightarrow_{\mathcal{B P}} \subseteq\left(P \times \Gamma^{*} \times 2^{\Delta \cup \Delta_{c}}\right) \times$ $2^{\left(P \times \Gamma^{*} \times 2^{\Delta \cup \Delta_{c}}\right)}$ between configurations as follows: Let $\theta \subseteq$ $\Delta \cup \Delta_{c}, \gamma \in \Gamma, w \in \Gamma^{*}$, and $p \in P$, then:

1) If $r:\langle p, \gamma\rangle \stackrel{\left[\sigma_{1}, \cdots, \sigma_{m}\right]}{\longrightarrow}\left\{\left\langle p_{1}, w_{1}\right\rangle, \cdots,\left\langle p_{m}, w_{m}\right\rangle\right\}$ is a rule in $\Delta \cap \theta$, if either for every $1 \leq i \leq$ $m, \sigma_{i}=-$ or $\exists 1 \leq i \leq m, \sigma_{i} \cap \theta \neq \emptyset$, then $(\langle p, \gamma w\rangle, \theta) \Rightarrow_{\mathcal{B P}}\left\{\left(\left\langle p_{i}, w_{i} w\right\rangle, \theta\right) \mid \sigma_{i}=-, 1 \leq i \leq\right.$ $m\} \cup\left\{\left(\left\langle p_{i}, w_{i} w\right\rangle, \theta\right) \mid \sigma_{i} \cap \theta \neq \emptyset, 1 \leq i \leq m\right\}$.

2) If $r: p \stackrel{\left(\sigma, \sigma^{\prime}\right)}{\longrightarrow} p^{\prime}$ is a rule in $\Delta_{c} \cap \theta$, then $(\langle p, w\rangle, \theta) \Rightarrow_{\mathcal{B P}}\left\{\left(\left\langle p^{\prime}, w\right\rangle, \theta^{\prime}\right)\right\}, \theta^{\prime}=\theta \backslash \sigma \cup \sigma^{\prime}$.

Intuitively, $\left[\sigma_{1}, \cdots, \sigma_{m}\right]$ in the transition $r$ : $\langle p, \gamma\rangle \stackrel{\left[\sigma_{1}, \cdots, \sigma_{m}\right]}{\longrightarrow}\left\{\left\langle p_{1}, w_{1}\right\rangle, \cdots,\left\langle p_{m}, w_{m}\right\rangle\right\}$ ensures that for a given configuration $(\langle p, \gamma w\rangle, \theta)$, for every $1 \leq i \leq n$, $\left(\left\langle p_{i}, w_{i} w\right\rangle, \theta\right)$ is in the set of immediate successor iff

- either for every $1 \leq j \leq n, \sigma_{j}=-$;

- or $\sigma_{i}=-$ and $\exists j \neq i, 1 \leq j \leq n$ s.t. $\sigma_{j} \cap \theta \neq \emptyset$

- or $\sigma_{i} \cap \theta \neq \emptyset$

Note that - means that there is no constraint on whether $\theta$ contains a rule in $\sigma_{i}$ or not. 
For every $c \in P \times \Gamma^{*} \times 2^{\Delta \cup \Delta_{c}}$ and $C \subseteq P \times \Gamma^{*} \times 2^{\Delta \cup \Delta_{c}}$, if $c \Rightarrow{ }_{\mathcal{B P}} C$ then $c$ is an immediate predecessor of $C$ and $C$ is an immediate successor of $c$. Let $\Rightarrow_{\mathcal{B} \mathcal{P}}^{*} \subseteq\left(P \times \Gamma^{*} \times\right.$ $\left.2^{\Delta \cup \Delta_{c}}\right) \times 2^{\left(P \times \Gamma^{*} \times 2^{\Delta \cup \Delta_{c}}\right)}$ be the reflexive transitive closure of $\Rightarrow_{\mathcal{B P}}$ defined as follows: (1) $\forall c \in P \times \Gamma^{*} \times 2^{\Delta \cup \Delta_{c}}$, $c \Rightarrow_{\mathcal{B P}}^{*}\{c\}$, (2) if $c \Rightarrow_{\mathcal{B P}} C$, then $c \Rightarrow_{\mathcal{B P}}^{*} C$, and (3) if $c \Rightarrow_{\mathcal{B P}}\left\{c_{1}, \ldots c_{n}\right\}$ and $c_{i} \Rightarrow_{\mathcal{B P}} C_{i}$ for every $1 \leq i \leq n$, then $c \Rightarrow_{\mathcal{B P}}^{*} \bigcup_{i=1}^{n} C_{i}$. Given a set of configurations $C$, we define the sets $\operatorname{pre}_{\mathcal{B P}}(C), \operatorname{pre}_{\mathcal{B} \mathcal{P}}^{*}(C)$ and $\operatorname{pre}_{\mathcal{B P}}^{+}(C)$ as follows: pre $_{\mathcal{B P}}(C)=\left\{c \in P \times \Gamma^{*} \times 2^{\Delta \cup \Delta_{c}} \mid \exists C^{\prime} \subseteq C\right.$ s.t. $C^{\prime}$ is an immediate successor of $c\}$, $\operatorname{pre}_{\mathcal{B P}}^{*}(C)=\left\{c \in P \times \Gamma^{*} \times\right.$ $2^{\Delta \cup \Delta_{c}}, \exists C^{\prime} \subseteq C$ s.t. $\left.c \Rightarrow_{\mathcal{B P}}^{*} C^{\prime}\right\}$ and $\operatorname{pre}_{\mathcal{B} \mathcal{P}}^{+}(C)=$ pre $_{\mathcal{B P}} \circ$ pre $_{\mathcal{B} \mathcal{P}}^{*}(C)$. We omit the subscript $\mathcal{B P}$ when it is clear from the context.

A run $\rho$ of $\mathcal{B P}$ starting from an initial configuration $c_{0}$ is a tree whose root is labelled by $c_{0}$ and whose other nodes are labelled by configurations of $P \times \Gamma^{*} \times 2^{\Delta \times \Delta_{c}}$. A node of $\rho$ labelled by configuration $c$ has $n$ children labelled by $c_{1}, \ldots, c_{n}$, respectively, iff $c \Rightarrow_{\mathcal{B P}}\left\{c_{1}, \ldots c_{n}\right\}$. A path $c_{0} c_{1} \cdots$ of a run $\rho$ is an infinite sequence of configurations s.t. $c_{0}$ is the root of $\rho$ and $c_{i+1}$ is one child of $c_{i}$. A path is accepting iff it visits some configurations with control locations in $F$ infinitely often. A run is accepting iff all its paths are accepting. A configuration $c$ is accepted by $\mathcal{B P}$ iff it is the root of a run accepted by $\mathcal{B P}$. The language of $\mathcal{B P}, L(\mathcal{B P})$, is the set of configurations accepted by $\mathcal{B P}$.

We assume w.l.o.g. that for every rule in $\Delta_{c}$ of the form $r: p \stackrel{\left(\sigma, \sigma^{\prime}\right)}{\longrightarrow} p^{\prime}, r \notin \sigma$.

\section{Representing potentially infinite sets of configurations of SM-ABPDSs.}

Alternating Multi-Automata (AMA) were introduced in [30] to finitely represent regular sets of configurations of an alternating PDS. In order to adapt AMA to represent regular sets of SM-ABPDS, we extend this notion taking phases into account as follows:

Definition 3. Let $\mathcal{B P}=\left(P, \Gamma, \Delta, \Delta_{c}, F\right)$ be a SM-ABPDS. An Extended Alternating Multi-Automaton (EAMA) is a tuple $\mathcal{A}=\left(Q, \Gamma, T, I, Q_{F}\right)$ where $I \subseteq P \times 2^{\Delta \cup \Delta_{c}} \subseteq Q$ is the set of initial states, $T \subseteq Q \times(\Gamma \cup\{\epsilon\}) \times 2^{Q}$ is the set of transitions, $Q_{F} \subseteq Q$ is a finite set of final states.

Let $\rightarrow_{T}$ be the transition relation defined as follows: (1) $\forall q \in Q, q \stackrel{\epsilon}{\rightarrow} T$ $\{q\}$ where $\epsilon$ is the empty word; (2) if $\left(q, \gamma,\left\{q_{1}, \cdots, q_{n}\right\}\right) \in T, q \stackrel{\gamma}{\rightarrow} T\left\{q_{1}, \cdots, q_{n}\right\}$; and (3) if $q \stackrel{\gamma}{\rightarrow}_{T}\left\{q_{1}, \cdots, q_{n}\right\}$ and $q_{i} \stackrel{w}{\rightarrow}_{T} Q_{i}$ for every $1 \leq i \leq n$, then $q \stackrel{\gamma w}{\longrightarrow} \bigcup_{i=1}^{n} Q_{i}$.

A configuration $(\langle p, w\rangle, \theta)$ is accepted by the EAMA $\mathcal{A}$ iff $(p, \theta) \in I$ and $\exists Q^{\prime} \subseteq Q_{F}$ such that $(p, \theta) \stackrel{w}{\longrightarrow} Q_{T}$. Let $L(\mathcal{A})$ be the set of configurations accepted by $\mathcal{A}$. Let $\mathcal{C}$ be a set of configurations of the SM-ABPDS $\mathcal{B P} . \mathcal{C}$ is regular if there exists an EAMA $\mathcal{A}$ such that $\mathcal{C}=L(\mathcal{A})$.
C. From CTL Model-Checking of SM-PDSs to the emptiness problem of SM-ABPDSs

Let $\mathcal{P}=\left(P, \Gamma, \Delta, \Delta_{c}\right)$ be a Self Modifying Pushdown System with an initial configuration $c_{0}=\left(\left\langle p_{0}, w_{0}\right\rangle, \theta_{0}\right)$. Given a set of atomic propositions $A t$, let $\nu: P \rightarrow 2^{A t}$ be a labeling function that associates each control location to a set of atomic propositions. Let $\varphi$ be a CTL formula over At. Our goal is to check whether $c_{0} \models_{\nu} \varphi$. This can be done by translating the SM-PDS into an equivalent PDS as described in Section 10 and in [I]], and then applying the standard CTL model-checking algorithm for PDSs [31]. However, as will be shown in the experiments section (Section $\nabla$ ), this approach is not efficient. Thus, we need a direct algorithm that operates directly on the SM-PDS without translating it into a PDS. We provide in this section a direct algorithm that performs CTL modelchecking on SM-PDSs. To this aim, we will compute a kind of product of the SM-PDS with $\varphi$ : we construct a Self Modifying Alternating Büchi Pushdown System $\mathcal{B P}_{\varphi}$ s.t. $\mathcal{B P}_{\varphi}$ accepts a configuration $c$ iff $c \models{ }_{\nu} \varphi$. Thus, determining whether $c_{0} \models{ }_{\nu} \varphi$ can be reduced to checking whether $c_{0} \in L\left(\mathcal{B P}_{\varphi}\right)$.

Let $\mathcal{B P} \mathcal{P}_{\varphi}=\left(P^{\prime}, \Gamma, \Delta^{\prime}, \Delta_{c}^{\prime}, F\right)$ be the SM-ABPDS defined as follows: $P^{\prime}=P \times c l(\varphi) \cup P^{c l(\varphi)}$, where $P^{c l(\varphi)}$ is the set of control locations in the form $p^{\psi}$ where $p \in P$ and $\psi \in \operatorname{cl}(\varphi)$, $F=\{[p, a] \mid a \in \operatorname{cl}(\varphi) \cap A t$ and $a \in \nu(p)\} \cup\{[p, \neg a] \mid \neg a \in$ $c l(\varphi), a \in A t$ and $a \notin \nu(p)\} \cup P \times c l_{\widetilde{U}}(\varphi)$ where $c l_{\widetilde{U}}(\varphi)$ is the set of formulae of $\operatorname{cl}(\varphi)$ in the form $E\left[\psi_{1} \widetilde{U} \psi_{2}\right]$ or $A\left[\psi_{1} \widetilde{U} \psi_{2}\right]$. In what follows, to compute $\Delta^{\prime}$ and $\Delta_{c}^{\prime}$, every rule $r \in \Delta \cup \Delta_{c}$ leads to a set of rules $\left\{r_{1}^{\prime}, \cdots, r_{n}^{\prime}\right\}$ of $\Delta^{\prime} \cup \Delta_{c}^{\prime}$, we call this set of rules $\operatorname{prod}(r)$. Moreover, let $\operatorname{prod}_{E}(r) \subseteq \operatorname{prod}(r)$ be the set of rules generated from $r$ using subformulas of the form $E X \psi_{1}, E\left[\psi_{1} U \psi_{2}\right]$ or $E\left[\psi_{1} \widetilde{U} \psi_{2}\right]$ ( see below for more details about $\operatorname{prod}(r)$ and $\left.\operatorname{prod}_{E}(r)\right)$.

The transition relations $\Delta^{\prime}$ and $\Delta_{c}^{\prime}$ (resp. the sets $\operatorname{prod}(r)$ and $\operatorname{prod}_{E}(r)$, for every $\left.r \in \Delta \cup \Delta_{c}\right)$ are the smallest sets of transitions (resp. of sets of rules) defined as follows: Initially, $\Delta^{\prime}=\Delta_{c}^{\prime}=\emptyset, \operatorname{prod}_{E}(r)=\emptyset$ and $\operatorname{prod}(r)=\emptyset, \forall r \in \Delta \cup \Delta_{c}$. $\forall p \in P, \forall \psi \in \operatorname{cl}(\varphi)$ and $\forall \gamma \in \Gamma$, we have:

1) if $\psi=a, a \in A t$ and $a \in \nu(p) ;\langle[p, a], \gamma\rangle \stackrel{[-]}{\longrightarrow}\langle[p, a], \gamma\rangle \in$ $\Delta^{\prime}$

2) if $\psi=\neg a, a \in A t$ and $a \notin \nu(p) ;\langle[p, \psi], \gamma\rangle \stackrel{[-]}{\longrightarrow}\langle[p, \psi], \gamma\rangle \in$ $\Delta^{\prime}$

3) if $\psi=\psi_{1} \vee \psi_{2} ;\langle[p, \psi], \gamma\rangle \stackrel{[-]}{\longrightarrow}\left\langle\left[p, \psi_{1}\right], \gamma\right\rangle \in \Delta^{\prime}$ and $\langle[p, \psi], \gamma\rangle \stackrel{[-]}{\longrightarrow}\left\langle\left[p, \psi_{2}\right], \gamma\right\rangle \in \Delta^{\prime}$

4) if $\psi=\psi_{1} \wedge \psi_{2} ;\langle[p, \psi], \gamma\rangle \stackrel{[-,-]}{\longrightarrow}\left\{\left\langle\left[p, \psi_{1}\right], \gamma\right\rangle,\left\langle\left[p, \psi_{2}\right], \gamma\right\rangle\right\} \in$ $\Delta^{\prime}$

5) if $\psi=E X \psi_{1}$, then:

a) if $p \in P_{N}$, for every $R=\langle p, \gamma\rangle \hookrightarrow\left\langle p^{\prime}, w\right\rangle \in \Delta, R^{\prime}=$ $\langle[p, \psi], \gamma\rangle \stackrel{[-]}{\longrightarrow}\left\langle\left[p^{\prime}, \psi_{1}\right], w\right\rangle \in \Delta^{\prime}, R^{\prime} \in \operatorname{prod}_{E}(R)$ and $R^{\prime} \in \operatorname{prod}(R)\left(R^{\prime} \in \operatorname{prod}(R)\right.$ means that $R^{\prime}$ is generated from $R$ and $R^{\prime} \in \operatorname{prod}_{E}(R)$ means that $R^{\prime}$ is generated from $R$ using a formula of the form $E X \psi_{1}$, $E\left[\psi_{1} U \psi_{2}\right]$ or $E\left[\psi_{1} \widetilde{U} \psi_{2}\right]$.)

b) if $p \in P_{C}$, for every $R=p \stackrel{\left(r_{1}, r_{2}\right)}{\longrightarrow} p^{\prime} \in \Delta_{c}, \quad R^{\prime}=$ $[p, \psi] \underset{\left(\sigma, \sigma^{\prime}\right)}{\longrightarrow}\left[p^{\prime}, \psi_{1}\right] \in \Delta_{c}^{\prime}$ where $\sigma=\operatorname{prod}\left(r_{1}\right), \sigma^{\prime}=$ $\operatorname{prod}\left(r_{2}\right), R^{\prime} \in \operatorname{prod}_{E}(R)$ and $R^{\prime} \in \operatorname{prod}(R)$

6) if $\psi=A X \psi_{1}$, then: 
a) if $p \in P_{N}$, let $\left\{R_{1}=\langle p, \gamma\rangle \hookrightarrow\left\langle p_{1}, w_{1}\right\rangle, \cdots, R_{n}=\right.$ $\left.\langle p, \gamma\rangle \hookrightarrow\left\langle p_{n}, w_{n}\right\rangle\right\}$ be the set of all the rules of $\Delta$ that have $\langle p, \gamma\rangle$ in the left-handside. Then, $\quad R^{\prime}=\langle[p, \psi], \gamma\rangle \stackrel{\left[\sigma_{1}, \cdots, \sigma_{n}\right]}{\longrightarrow}$ $\left\{\left\langle\left[p_{1}, \psi_{1}\right], w_{1}\right\rangle, \cdots,\left\langle\left[p_{n}, \psi_{1}\right], w_{n}\right\rangle\right\} \in \Delta^{\prime}$, where for every $1 \leq i \leq n, \sigma_{i}=\operatorname{prod}_{E}\left(R_{i}\right)$ and $R^{\prime} \in \operatorname{prod}\left(R_{i}\right)$.

b) if $p \in P_{C}$, let $\left\{R_{1}=p \stackrel{\left(r_{1}, r_{1}^{\prime}\right)}{\longrightarrow} p_{1}, \cdots, R_{n}=\right.$ $\left.p \stackrel{\left(r_{n}, r_{n}^{\prime}\right)}{\longrightarrow} p_{n}\right\}$ be the set of all the rules of $\Delta_{c}$ that have $p$ in the left-hand-side. Then, for every $\gamma \in \Gamma, R_{\perp}^{\prime}=$ $\langle[p, \psi], \gamma\rangle \stackrel{\left[\sigma_{1}, \cdots, \sigma_{n}\right]}{\longrightarrow}\left\{\left\langle p_{1}^{\psi}, \gamma\right\rangle, \cdots,\left\langle p_{n}^{\psi}, \gamma\right\rangle\right\} \in \Delta^{\prime}$ and for every $1 \leq i \leq n, R_{i}^{\prime}: p_{i}^{\psi} \stackrel{\left(\sigma, \sigma^{\prime}\right)}{\longrightarrow}\left[p_{i}, \psi_{1}\right] \in \Delta_{c}^{\prime}$, where for every $1 \leq i \leq n, \sigma_{i}=\operatorname{prod}_{E}\left(R_{i}\right)$, $\sigma=\operatorname{prod}\left(r_{i}\right), \sigma^{\prime}=\operatorname{prod}\left(r_{i}^{\prime}\right)$, and for every $1 \leq i \leq n$, $R_{\perp}^{\prime}, R^{\prime} \in \operatorname{prod}\left(R_{i}\right)$.

7) if $\psi=E\left[\psi_{1} U \psi_{2}\right]$, then $\langle[p, \psi], \gamma\rangle \stackrel{[-]}{\longrightarrow}\left\langle\left[p, \psi_{2}\right], \gamma\right\rangle \in \Delta^{\prime}$ and:

a) if $p \in P_{N}$, for every $R=\langle p, \gamma\rangle \hookrightarrow\left\langle p^{\prime}, w\right\rangle \in \Delta$,

$R^{\prime}=\langle[p, \psi], \gamma\rangle \stackrel{[-,-]}{\longrightarrow}\left\{\left\langle\left[p, \psi_{1}\right], \gamma\right\rangle,\left\langle\left[p^{\prime}, \psi\right], w\right\rangle\right\} \in \Delta^{\prime}$, $R^{\prime} \in \operatorname{prod}_{E}(R)$ and $R^{\prime} \in \operatorname{prod}(R)$.

b) if $p \in P_{C}$, for every $R=p \stackrel{\left(r_{1}, r_{1}^{\prime}\right)}{\longrightarrow} p^{\prime} \in \Delta_{c}$, then for every $\gamma \in \Gamma, R_{\perp}^{\prime}=\langle[p, \psi], \gamma\rangle \stackrel{[-,-]}{\longrightarrow}$ $\left\{\left\langle\left[p, \psi_{1}\right], \gamma\right\rangle,\left\langle p^{\psi}, \gamma\right\rangle\right\} \in \Delta^{\prime}$ and $p^{\psi} \stackrel{\left(\sigma, \sigma^{\prime}\right)}{\longrightarrow}\left[p^{\prime}, \psi\right] \in \Delta_{c}^{\prime}$ where $\sigma=\operatorname{prod}\left(r_{1}\right), \sigma^{\prime}=\operatorname{prod}\left(r_{1}^{\prime}\right), \quad R_{\perp}^{\prime}, R^{\prime} \in$ $\operatorname{prod}_{E}(R)$ and $R_{\perp}^{\prime}, R^{\prime} \in \operatorname{prod}(R)$.

8) if $\psi=A\left[\psi_{1} U \psi_{2}\right]$, then $\langle[p, \psi], \gamma\rangle \stackrel{[-]}{\longrightarrow}\left\langle\left[p, \psi_{2}\right], \gamma\right\rangle \in \Delta^{\prime}$, and:

a) if $p \in P_{N}$, let $\left\{R_{1}=\langle p, \gamma\rangle \hookrightarrow\left\langle p_{1}, w_{1}\right\rangle, \cdots, R_{n}=\right.$ $\left.\langle p, \gamma\rangle \hookrightarrow\left\langle p_{n}, w_{n}\right\rangle\right\}$ be the set of all the rules of $\Delta$ that have $\langle p, \gamma\rangle$ in the left-handside. Then, $R^{\prime}=\langle[p, \psi], \gamma\rangle \stackrel{\left[-, \sigma_{1}, \cdots, \sigma_{n}\right]}{\stackrel{\left[-\omega_{n}\right.}{\longrightarrow}}$ $\left\{\left\langle\left[p, \psi_{1}\right], \gamma\right\rangle,\left\langle\left[p_{1}, \psi\right], w_{1}\right\rangle, \cdots,\left\langle\left[p_{n}, \psi\right], w_{n}\right\rangle\right\} \in \Delta^{\prime}$ where for every $1 \leq i \leq n, \sigma_{i}=\operatorname{prod}_{E}\left(R_{i}\right)$, and $R^{\prime} \in \operatorname{prod}\left(R_{i}\right)$.

b) if $p \in P_{C}$, let $\left\{R_{1}=p \stackrel{\left(r_{1}, r_{1}^{\prime}\right)}{\longrightarrow} p_{1}, \cdots, R_{n}=\right.$ $\left.p \stackrel{\left(r_{n}, r_{n}^{\prime}\right)}{\longrightarrow} p_{n}\right\}$ be the set of all the rules of $\Delta_{c}$ that have $p$ in the left-hand-side. Then, $\forall 1 \leq i \leq n$, for every $\gamma \in \Gamma, R_{\perp}:\langle[p, \psi], \gamma\rangle \stackrel{\left[-, \sigma_{1}, \cdots, \sigma_{n}\right]}{\longrightarrow}$ $\left\{\left\langle\left[p, \psi_{1}\right], \gamma\right\rangle,\left\langle p_{1}^{\psi}, \gamma\right\rangle, \cdots,\left\langle p_{n}^{\psi}, \gamma\right\rangle\right\} \in \Delta^{\prime}$ and $R_{i}^{\prime}$ : $p_{i}^{\psi} \stackrel{\left(\sigma, \sigma^{\prime}\right)}{\longrightarrow}\left[p_{i}, \psi\right] \in \Delta_{c}^{\prime}$ where for every $1 \leq i \leq$ $n, \sigma_{i}=\operatorname{prod}_{E}\left(R_{i}\right), \sigma=\operatorname{prod}\left(r_{i}\right), \sigma^{\prime}=\operatorname{prod}\left(\overline{r_{i}^{\prime}}\right)$ and $R_{\perp}, R_{i}^{\prime} \in \operatorname{prod}\left(R_{i}\right)$.

9) if $\psi=E\left[\psi_{1} \widetilde{U} \psi_{2}\right]$, then $\langle[p, \psi], \gamma\rangle \stackrel{[-,-]}{\longrightarrow}$ $\left\{\left\langle\left[p, \psi_{2}\right], \gamma\right\rangle,\left\langle\left[p, \psi_{1}\right], \gamma\right\rangle\right\} \in \Delta^{\prime}$ and:

a) if $p \in P_{N}$, then for every $R=\langle p, \gamma\rangle \hookrightarrow\left\langle p^{\prime}, w\right\rangle \in \Delta$, $\left.R^{\prime}=\langle[p, \psi], \gamma\rangle \stackrel{[-,-]}{\longrightarrow}\left\{\left[p, \psi_{2}\right], \gamma\right\rangle,\left\langle\left[p^{\prime}, \psi\right], w\right\rangle\right\} \in \Delta^{\prime}$, $R^{\prime} \in \operatorname{prod}_{E}(R)$ and $R^{\prime} \in \operatorname{prod}(R)$.

b) if $p \in P_{C}$, then for every $R=p \stackrel{\left(r_{1}, r_{1}^{\prime}\right)}{\longleftrightarrow} p^{\prime} \in$ $\Delta_{c}$, for every $\gamma \in \Gamma, R_{\perp}=\langle[p, \psi], \gamma\rangle \stackrel{[-,-]}{\stackrel{\left[\left(\rho^{\prime}\right)\right.}{\longrightarrow}}$ $\left\{\left\langle\left[p, \psi_{2}\right], \gamma\right\rangle,\left\langle p^{\psi}, \gamma\right\rangle\right\} \in \Delta^{\prime}$ and $R^{\prime}: p^{\psi} \stackrel{\left(\sigma, \sigma^{\prime}\right)}{\longrightarrow}$ $\left[p^{\prime}, \psi\right] \in \Delta_{c}^{\prime}$ where $\sigma=\operatorname{prod}\left(r_{1}\right), \sigma^{\prime}=\operatorname{prod}\left(r_{1}^{\prime}\right)$, $R_{\perp}, R^{\prime} \in \operatorname{prod}_{E}(R)$ and $R_{\perp}, R^{\prime} \in \operatorname{prod}(R)$.

$10)$ if $\psi=A\left[\psi_{1} \widetilde{U} \psi_{2}\right]$, then $\langle[p, \psi], \gamma\rangle \stackrel{[-,-]}{\longrightarrow}$ $\left\{\left\langle\left[p, \psi_{2}\right], \gamma\right\rangle,\left\langle\left[p, \psi_{1}\right], \gamma\right\rangle\right\} \in \Delta^{\prime}$, and:

a) if $p \in P_{N}$, let $\left\{R_{1}=\langle p, \gamma\rangle \hookrightarrow\left\langle p_{1}, w_{1}\right\rangle, \cdots, R_{n}=\right.$ $\left.\langle p, \gamma\rangle \hookrightarrow\left\langle p_{n}, w_{n}\right\rangle\right\}$ be the set of all the rules of $\Delta$ that have $\langle p, \gamma\rangle$ in the left-hand-side. Then for every $1 \leq$ $i \leq n, \sigma_{i}=\operatorname{prod}_{E}\left(R_{i}\right), R^{\prime}=\langle[p, \psi], \gamma\rangle \stackrel{\left[-, \sigma_{1}, \cdots, \sigma_{n}\right]}{\longrightarrow}$ $\left\{\left\langle\left[p, \psi_{2}\right], \gamma\right\rangle,\left\langle\left[p_{1}, \psi\right], w_{1}\right\rangle, \cdots,\left\langle\left[p_{n}, \psi\right], w_{n}\right\rangle\right\} \in \Delta^{\prime}$ and $R^{\prime} \in \operatorname{prod}\left(R_{i}\right)$.

b) if $p \in P_{C}$, let $\left\{R_{1}=p \stackrel{\left(r_{1}, r_{1}^{\prime}\right)}{\longrightarrow} p_{1}, \cdots, R_{n}=\right.$ $\left.p \stackrel{\left(r_{n}, r_{n}^{\prime}\right)}{\longrightarrow} p_{n}\right\}$ be the set of all the rules of $\Delta_{c}$ that have $p$ in the left-hand-side. Then, for every $\gamma \in \Gamma, R_{\perp}=\langle[p, \psi], \gamma\rangle \stackrel{\left[-, \sigma_{1}, \cdots, \sigma_{n}\right]}{\longrightarrow}$ $\left\{\left\langle\left[p, \psi_{2}\right], \gamma\right\rangle,\left\langle p_{1}^{\psi}, \gamma\right\rangle, \cdots,\left\langle p_{n}^{\psi}, \gamma\right\rangle\right\} \in \Delta^{\prime}, \forall 1 \leq i \leq$ $n, \sigma_{i}=\operatorname{prod}_{E}\left(R_{i}\right)$ and for every $1 \leq i \leq n, R_{i}^{\prime}$ : $p_{i}^{\psi} \stackrel{\left(\sigma, \sigma^{\prime}\right)}{\longrightarrow}\left[p_{i}, \psi\right] \in \Delta_{c}^{\prime}$ where $\sigma=\operatorname{prod}\left(r_{i}\right), \sigma^{\prime}=$ $\operatorname{prod}\left(r_{i}^{\prime}\right)$ and $R_{\perp}, R_{i}^{\prime} \in \operatorname{prod}\left(R_{i}\right)$.

Let $\operatorname{prod}(\Delta)=\left\{r^{\prime} \in \Delta^{\prime} \mid \exists r \in \Delta, r^{\prime} \in \operatorname{prod}(r)\right\}$ be the set of rules of $\Delta^{\prime}$ that are generated from $\Delta$. Let $\delta=\Delta^{\prime} \backslash \operatorname{prod}(\Delta)$ be the set of rules of $\Delta^{\prime}$ that are not generated from any rule of $\Delta$ nor $\Delta_{c}$ (e.g., the rules computed by items $1,2,3$ and 4 are in $\delta$ ). These rules $\delta$ are independent of $\Delta$ and $\Delta_{c}$. They are introduced by the structure of $\varphi$. Thus, they need to be present in all the phases of $\mathcal{B P}{ }_{\varphi}$. Let then $\theta \subseteq \Delta \cup \Delta_{c}$ be a phase of $\mathcal{P}$. Its corresponding phase in $\mathcal{B P}_{\varphi}$ is $\beta(\theta)=\operatorname{prod}(\theta) \cup \delta$, where $\operatorname{prod}(\theta)=\left\{r^{\prime} \in \Delta^{\prime} \cup \Delta_{c}^{\prime} \mid \exists r \in \theta, r^{\prime} \in \operatorname{prod}(r)\right\}$.

Let us explain the above construction intuitively. The above automaton $\mathcal{B P}_{\varphi}$ can be seen as a kind of product of the SM$\operatorname{PDS} \mathcal{P}$ with the formula $\varphi$. For $\psi \in \operatorname{cl}(\varphi),(\langle p, w\rangle, \theta) \mid{ }_{\nu} \psi$ iff $\mathcal{B P}_{\varphi}$ accepts a configuration $(\langle[p, \psi], w\rangle, \beta(\theta))$. We give in what follows the intuition behind all the items above:

If $\psi=a \in A t$, then $\forall w \in \Gamma^{*}, \theta \subseteq \Delta \cup \Delta_{c},(\langle p, w\rangle, \theta) \models{ }_{\nu} \psi$ iff $a \in \nu(p)$. Hence, the automaton $\mathcal{B P} \varphi$ should accept a run starting from $(\langle[p, a], w\rangle, \beta(\theta))$ iff $a \in \nu(p) .[p, a] \in F$ iff $a \in \nu(p)$. Thus, the loop added in $(\langle[p, a], w\rangle, \beta(\theta))$ by Item 1 makes sure that $\mathcal{B P}_{\varphi}$ accepts this run.

If $\psi=\neg a$, then $\forall w \in \Gamma^{*}, \theta \subseteq \Delta \cup \Delta_{c},(\langle p, w\rangle, \theta) \models{ }_{\nu} \psi$ iff $a \notin \nu(p)$. Hence, the automaton $\mathcal{B P}_{\varphi}$ should accept a run starting from $(\langle[p, \neg a], w\rangle, \beta(\theta))$ iff $a \notin \nu(p) .[p, \neg a] \in F$ iff $a \notin \nu(p)$. Thus, the loop in $(\langle[p, \neg a], w\rangle, \beta(\theta))$ added by Item 2 ensures that $\mathcal{B P}_{\varphi}$ accepts this run.

If $\psi=\psi_{1} \vee \psi_{2}$, then $\forall w \in \Gamma^{*}, \theta \subseteq \Delta \cup \Delta_{c},(\langle p, w\rangle, \theta) \mid=_{\nu} \psi$ iff $\left((\langle p, w\rangle, \theta) \models{ }_{\nu} \psi_{1}\right.$ or $\left.(\langle p, w\rangle, \theta) \mid=_{\nu} \psi_{2}\right)$. Thus, $\mathcal{B P}_{\varphi}$ accepts a run starting from $\left(\left\langle\left[p, \psi_{1} \vee \psi_{2}\right], w\right\rangle, \beta(\theta)\right)$ iff $\mathcal{B P}_{\varphi}$ has an accepting run starting from $\left(\left\langle\left[p, \psi_{1}\right], w\right\rangle, \beta(\theta)\right)$ or $\left(\left\langle\left[p, \psi_{2}\right], w\right\rangle, \beta(\theta)\right)$. This is ensured by Item 3 . Item 4 is similar to Item 3 , it handles the case $\psi=\psi_{1} \wedge \psi_{2}$ where $(\langle p, w\rangle, \theta)$ satisfies $\psi$ iff it satisfies both $\psi_{1}$ and $\psi_{2}$.

If $\psi=E X \psi_{1}$, then $\forall w \in \Gamma^{*}, \theta \subseteq \Delta \cup \Delta_{c},(\langle p, w\rangle, \theta) \models{ }_{\nu} \psi$ iff an immediate successor $\left(\left\langle p^{\prime}, w^{\prime}\right\rangle, \theta^{\prime}\right)$ of $(\langle p, w\rangle, \theta)$ satisfies $\psi_{1}$. Thus, $\mathcal{B P}_{\varphi}$ has an accepting run from $(\langle[p, \psi], w\rangle, \beta(\theta))$ iff it can accept a run from $\left(\left\langle\left[p^{\prime}, \psi_{1}\right], w^{\prime}\right\rangle, \beta\left(\theta^{\prime}\right)\right)$. There are two cases depending on whether $p \in P_{N}$ or $p \in P_{c}$, because the form of the rules of the SM-PDS depends on whether $p \in$ $P_{N}$ or $p \in P_{c}$ : if $p \in P_{N}$, then necessarily, the rules that can be applied from $p$ are of the form $\langle p, \gamma\rangle \hookrightarrow\left\langle p^{\prime}, w\right\rangle \in \Delta$, whereas if $p \in P_{c}$, then necessarily, the rules that can be applied from $p$ are of the form $r: p \stackrel{\left(r_{1}, r_{2}\right)}{\longrightarrow} p^{\prime} \in \Delta_{c}$. Thus, if $p \in P_{N}$, then $\mathcal{B P}_{\varphi}$ has an accepting run from $(\langle[p, \psi], \gamma u\rangle, \beta(\theta))$ iff there exists a rule $\langle p, \gamma\rangle \hookrightarrow\left\langle p^{\prime}, w\right\rangle \in \Delta$ such that $\mathcal{B P} \mathcal{P}_{\varphi}$ has an accepting run from $\left(\left\langle\left[p^{\prime}, \psi_{1}\right], w u\right\rangle, \beta(\theta)\right)$. This is ensured by Item 5(a). If $p \in P_{c}$, then $\mathcal{B P}_{\varphi}$ has an accepting run 
from $(\langle[p, \psi], \gamma u\rangle, \beta(\theta))$ iff there exists a rule $r: p \stackrel{\left(r_{1}, r_{2}\right)}{\longrightarrow}$ $p^{\prime} \in \Delta_{c} \cap \theta$ such that $\mathcal{B P}_{\varphi}$ has an accepting run from $\left(\left\langle\left[p^{\prime}, \psi_{1}\right], \gamma u\right\rangle, \beta\left(\theta^{\prime}\right)\right)$, where $\theta^{\prime}=\left(\theta \backslash\left\{r_{1}\right\}\right) \cup\left\{r_{2}\right\}$. This is ensured by Item $5(\mathrm{~b})$.

If $\psi=A X \psi_{1}$, then $\forall w \in \Gamma^{*}, \theta \subseteq \Delta \cup \Delta_{c}$, $(\langle p, w\rangle, \theta) \models{ }_{\nu} \psi$ iff every immediate successor $\left(\left\langle p^{\prime}, w^{\prime}\right\rangle, \theta^{\prime}\right)$ of $(\langle p, w\rangle, \theta)$ satisfies $\psi_{1}$. Thus, $\mathcal{B P}_{\varphi}$ has an accepting run from $(\langle[p, \psi], w\rangle, \beta(\theta))$ iff it can accept a run from all its immediate successors $\left(\left\langle\left[p^{\prime}, \psi_{1}\right], w^{\prime}\right\rangle, \beta\left(\theta^{\prime}\right)\right)$. As previously, there are two cases depending on whether $p \in P_{N}$ or $p \in P_{c}$ : if $p \in P_{N}$, let $\gamma \in \Gamma$ and $u \in \Gamma^{*}$ such that $w=\gamma u$. Let then $\left\{\langle p, \gamma\rangle \hookrightarrow\left\langle p_{1}, w_{1}\right\rangle, \cdots,\langle p, \gamma\rangle \hookrightarrow\left\langle p_{m}, w_{m}\right\rangle\right\}$ be the set of all the rules of $\Delta \cap \theta$ that have $\langle p, \gamma\rangle$ in the left-hand-side. Then, $\mathcal{B P}_{\varphi}$ has an accepting run from $(\langle[p, \psi], \gamma u\rangle, \beta(\theta))$ iff $\mathcal{B P}_{\varphi}$ has an accepting run from every $\left(\left\langle\left[p_{i}, \psi_{1}\right], w_{i} u\right\rangle, \beta(\theta)\right)$, $1 \leq i \leq m$. This is ensured by Item 6(a). Note that Item 6(a) considers all the rules $R_{i}:\langle p, \gamma\rangle \hookrightarrow\left\langle p_{i}, w_{i}\right\rangle$ that are in $\Delta$ (even those that are not in $\theta$ ), then the constraints $\left[\sigma_{1}, \cdots, \sigma_{n}\right]$ of the rule $R^{\prime}$ of Item 6(a) ensures that only the $R_{i}$ 's that are in $\theta$ are applied. Note also that in $R^{\prime}, \sigma_{i}=\operatorname{prod}_{E}\left(R_{i}\right)$ ensures that $\sigma_{i} \cap \beta(\theta) \neq \emptyset$ iff $R_{i} \cap \theta \neq \emptyset$. Here taking $\sigma_{i}=\operatorname{prod}\left(R_{i}\right)$ is not correct because $R^{\prime} \in \operatorname{prod}\left(R_{i}\right)$ and so in this case, $\sigma_{i} \cap \beta(\theta)$ would always be nonempty. On the other hand, if $p \in P_{c}$, let $\left\{p \stackrel{\left(r_{1}, r_{1}^{\prime}\right)}{\longrightarrow} p_{1}, \cdots, p \stackrel{\left(r_{m}, r_{m}^{\prime}\right)}{\longrightarrow} p_{m}\right\}$ be the set of all the rules of $\Delta_{c} \cap \theta$ that have $p$ in the left-handside. Then $\mathcal{B P} \mathcal{P}_{\varphi}$ has an accepting run from $(\langle[p, \psi], \gamma u\rangle, \beta(\theta))$ iff $\mathcal{B P} \mathcal{P}_{\varphi}$ has an accepting run from $\left(\left\langle\left[p_{i}, \psi_{1}\right], \gamma u\right\rangle, \beta\left(\theta_{i}\right)\right)$, for every $1 \leq i \leq m$, where $\theta_{i}=\left(\theta \backslash\left\{r_{i}\right\}\right) \cup\left\{r_{i}^{\prime}\right\}$. This is ensured by Item 6(b). As previously, Item 6(b) considers all the rules $R_{i}: p \stackrel{\left(r_{i}, r_{i}^{\prime}\right)}{\longrightarrow} p_{i}$ that are in $\Delta_{c}$ (even those that are not in $\theta)$, then the constraints $\left[\sigma_{1}, \cdots, \sigma_{n}\right]$ of the rule $R_{\perp}^{\prime}=\langle[p, \psi], \gamma\rangle \stackrel{\left[\sigma_{1}, \cdots, \sigma_{n}\right]}{\longrightarrow}\left\{\left\langle p_{1}^{\psi}, \gamma\right\rangle, \cdots,\left\langle p_{n}^{\psi}, \gamma\right\rangle\right\}$ of Item $6(\mathrm{~b})$ ensures that only the $R_{i}$ 's that are in $\theta$ are applied. Then $R_{i}^{\prime}: p_{i}^{\psi} \stackrel{\left(\sigma, \sigma^{\prime}\right)}{\longrightarrow}\left[p_{i}, \psi_{1}\right]$ ensures $\mathcal{B P} \varphi$ has an accepting run from $\left(\left\langle p_{i}^{\psi}, \gamma u\right\rangle, \beta(\theta)\right)$ iff $\mathcal{B P} \mathcal{P}_{\varphi}$ has an accepting run from $\left(\left\langle\left[p_{i}, \psi_{1}\right], \gamma u\right\rangle, \beta\left(\theta_{i}\right)\right)$ where $\theta_{i}=\left(\theta \backslash\left\{r_{i}\right\}\right) \cup\left\{r_{i}{ }^{\prime}\right\}$ for $1 \leq i \leq n$. Note that $\sigma^{\prime}=\operatorname{prod}\left(r_{i}\right)$ and $\sigma^{\prime}=\operatorname{prod}\left(r_{i}^{\prime}\right)$, then $\beta\left(\theta_{i}\right)=\beta(\theta) \backslash \sigma \cup \sigma^{\prime}$. Thus, $\mathcal{B P}_{\varphi}$ has an accepting run from $(\langle[p, \psi], \gamma u\rangle, \beta(\theta))$ iff $\mathcal{B P}_{\varphi}$ has an accepting run from $\left(\left\langle\left[p_{i}, \psi_{1}\right], \gamma u\right\rangle, \beta\left(\theta_{i}\right)\right)$ for every $1 \leq i \leq n$.

If $\psi=E\left[\psi_{1} U \psi_{2}\right]$, then $\forall w \in \Gamma^{*}, \theta \subseteq \Delta \cup \Delta_{c}$, $(\langle p, w\rangle, \theta) \models{ }_{\nu} \psi$ iff either it satisfies $\psi_{2}$ or it satisfies $\psi_{1}$ and there exists an immediate successor satisfying $\psi$. Thus, $\mathcal{B P}_{\varphi}$ has an accepting run from $(\langle[p, \psi], w\rangle, \beta(\theta))$ iff:

1) $\mathcal{B P}_{\varphi}$ has an accepting run from $\left(\left\langle\left[p, \psi_{2}\right], w\right\rangle, \beta(\theta)\right)$. This is handled by the rules $\langle[p, \psi], \gamma\rangle$ $\stackrel{[-,-]}{\longrightarrow}\left\{\left\langle\left[p, \psi_{2}\right], \gamma\right\rangle,\left\langle\left[p, \psi_{1}\right], \gamma\right\rangle\right\}$ introduced by Item 7 .

2) or $\mathcal{B P}_{\varphi}$ has an accepting run from both $\left(\left\langle\left[p, \psi_{1}\right], w\right\rangle, \beta(\theta)\right)$ and

$\left(\left\langle\left[p^{\prime}, \psi\right], w^{\prime}\right\rangle, \beta\left(\theta^{\prime}\right)\right)$ where $\left(\left\langle p^{\prime}, w^{\prime}\right\rangle, \theta^{\prime}\right)$ is an immediate successor of $(\langle p, w\rangle, \theta))$. There are two cases depending on whether $p \in P_{N}$ or $p \in P_{C}$ : the case $p \in P_{N}$ is handled by Item 7(a). Its intuition is similar to the intuition behind the previous items. Let then $p \in P_{C}$. Then there exists a rule $r: p \stackrel{\left(r_{1}, r_{1}^{\prime}\right)}{\longrightarrow} p^{\prime} \in \theta \cap \Delta_{c}$ such that $\mathcal{B P} \mathcal{P}_{\varphi}$ has an accepting run from both $\left(\left\langle\left[p, \psi_{1}\right], w\right\rangle, \beta(\theta)\right)$ and $\left(\left\langle\left[p^{\prime}, \psi\right], w\right\rangle, \beta\left(\theta^{\prime}\right)\right)$, where $\theta^{\prime}=\theta \backslash\left\{r_{1}\right) \cup\left\{r_{1}^{\prime}\right\}$. This is ensured by the rule $R_{\perp}=\langle[p, \psi], \gamma\rangle \stackrel{[-,-]}{\longrightarrow}$ $\left\{\left\langle\left[p, \psi_{2}\right], \gamma\right\rangle,\left\langle p^{\psi}, \gamma\right\rangle\right\} \in \Delta^{\prime}$ and $R^{\prime}: p^{\psi} \stackrel{\left(\sigma, \sigma^{\prime}\right)}{\longrightarrow}\left[p^{\prime}, \psi\right] \in$ $\Delta_{c}^{\prime}$ added by Item 7(b).

The case $\psi=A\left[\psi_{1} U \psi_{2}\right]$ is handled in a similar way using Items 8. If $\psi=E\left[\psi_{1} \widetilde{U} \psi_{2}\right]$, then $\forall w \in \Gamma^{*}, \theta \subseteq \Delta \cup \Delta_{c}$, $(\langle p, w\rangle, \theta) \mid{ }_{\nu} \quad \psi$ iff it satisfies $\psi_{2}$ and either it satisfies also $\psi_{1}$, or it has a successor $\left(\left\langle p^{\prime}, w^{\prime}\right\rangle, \theta^{\prime}\right)$ that satisfies $\psi$. Then, $\mathcal{B P}_{\varphi}$ has an accepting run from $(\langle[p, \psi], w\rangle, \theta)$ iff $\mathcal{B P} \mathcal{P}_{\varphi}$ has an accepting run from both $\left(\left(\left\langle\left[p, \psi_{2}\right], w\right\rangle, \beta(\theta)\right)\right.$ and $\left.\left(\left\langle\left[p, \psi_{1}\right], w\right\rangle, \beta(\theta)\right)\right)$, or it has an accepting run from both $\left(\left(\left\langle\left[p, \psi_{2}\right], w\right\rangle, \beta(\theta)\right)\right.$ and $\left.\left(\left\langle\left[p^{\prime}, \psi\right], w^{\prime}\right\rangle, \beta\left(\theta^{\prime}\right)\right)\right)$. This case is handled by Items 9 . To ensure that the runs on which $\psi_{2}$ always holds are accepted, we add $[p, \psi]$ in $F$. The case where $\psi=A\left[\psi_{1} \widetilde{U} \psi_{2}\right]$ is handled similarly by Items 10 .

We can show that (the proof is in the full version [14]):

Theorem 1. Let $(\langle p, w\rangle, \theta)$ be a configuration of the SM$\operatorname{PDS} \mathcal{P}$. $(\langle p, w\rangle, \theta) \models{ }_{\nu} \varphi$ iff $\mathcal{B} \mathcal{P} \varphi$ has an accepting run from $(\langle[p, \varphi], w\rangle, \beta(\theta))$.

Therefore, CTL model-checking for SM-PDSs can be reduced to the problem of determining whether a SM-ABPDS has an accepting run (emptiness analysis).

\section{Computing the language of a SM-ABPDS}

From now on, we fix a SM-ABPDS $\mathcal{B P}=\left(P, \Delta, \Delta_{c}, \Gamma, F\right)$. We show in this section that the set of configurations accepted by $\mathcal{B P}$ is regular and can be effectively represented by an EAMA (extended Alternating Multi-automaton). To this aim, we first characterize the set of configurations $L(\mathcal{B P})$ from which $\mathcal{B P}$ has an accepting run. Then we use this characterization to compute an EAMA that accepts it.

\section{A. Characterizing $L(\mathcal{B P})$}

Let $\left(X_{i}\right)_{i \geq 0}$ be the following sequence: $X_{0}=P \times \Gamma^{*} \times$ $2^{\Delta \cup \Delta_{c}}$, and for every $i \geq 0, X_{i+1}=\operatorname{Pre}_{\mathcal{B} \mathcal{P}}^{+}\left(X_{i} \cap\left(F \times \Gamma^{*} \times\right.\right.$ $\left.\left.2^{\Delta \cup \Delta_{c}}\right)\right)$. Let $Y_{\mathcal{B P}}=\bigcap_{i \geq 0} X_{i}$. We can show that $L(\mathcal{B P})=$ $Y_{\mathcal{B P}}$ :

Theorem 2. A SM-ABPDS $\mathcal{B P}$ has an accepting run starting from a configuration $(\langle p, w\rangle, \theta)$ iff $(\langle p, w\rangle, \theta) \in Y_{\mathcal{B P}}$.

Proving this theorem is based on the following lemma:

Lemma 1. $\mathcal{B P}$ has a run $\rho$ starting from a configuration $(\langle p, w\rangle, \theta)$ s.t. each path of $\rho$ visits configurations with control locations in $F$ at least $k$ times iff $(\langle p, w\rangle, \theta) \in X_{k}$.

Indeed, let $c \in X_{1}$. Then $c$ has a successor $C \subseteq F \times$ $\Gamma^{*} \times 2^{\Delta \cup \Delta_{c}}\left(\right.$ since $X_{1}=\operatorname{Pre}_{\mathcal{B} \mathcal{P}}^{+}\left(X_{0} \cap\left(F \times \Gamma^{*} \times 2^{\Delta \cup \Delta_{c}}\right)\right)$ ). Therefore, $\mathcal{B P}$ has a run starting from $c$ that visits some configuration $p \in F$ at least once. $X_{2}=\operatorname{Pre}_{\mathcal{B} \mathcal{P}}^{+}\left(X_{1} \cap(F \times\right.$ $\left.\Gamma^{*} \times 2^{\Delta \cup \Delta_{c}}\right)$ ), thus $\forall c^{\prime} \in X_{2}$, a run starting from $c^{\prime}$ will visit configurations in $X_{1} \cap\left(F \times \Gamma^{*} \times 2^{\Delta \cup \Delta_{c}}\right)$ at least once; and thus, it visits configurations with control locations in $F$ at least 
twice. Thus, we can get by induction that $\forall k \geq 1$, for every configuration $c$ in $X_{k}, \mathcal{B P}$ has a run that visits configurations with control locations in $F$ at least $k$ times.

\section{B. Computing $Y_{\mathcal{B P}}$}

In this section, our goal is to compute $Y_{\mathcal{B P}}$. We show that this set can be effectively represented by an EAMA $\mathcal{A}=\left(Q, \Gamma, T, I, Q_{f}\right)$, where $Q \subseteq P \times 2^{\Delta \cup \Delta_{c}} \times \mathbb{N} \cup\left\{q_{f}\right\}$, $I \subseteq P \times 2^{\Delta \cup \Delta_{c}} \times \mathbb{N}$ is the set of initial states and $q_{f}$ is the final state $\left(Q_{f}=\left\{q_{f}\right\}\right)$. Following [13], we propose a saturation procedure to compute $\mathcal{A}$ iteratively. Algorithm 1 below computes $\mathcal{A}$. Intuitively, it computes the different $X_{i}$ 's iteratively. Each iteration step $i$ computes an EAMA $\mathcal{A}_{i}$. States of $\mathcal{A}_{i}$ are of the form $(p, \theta)^{i}$, where $p \in P$ and $\theta \subseteq \Delta \cup \Delta_{c}$. There are two loops in the algorithm: the outer loop $\left(l o o p_{1}\right)$ and the inner loop $\left(l o o p_{2}\right)$. As will be explained later, if the sequence $\left(X_{i}\right)$ is strictly decreasing, the outer loop won't terminate. So we introduce two projections to force termination as follows: for every $S \subseteq P \times 2^{\Delta \cup \Delta_{c}} \times \mathbb{N} \cup\left\{q_{f}\right\}$ :

$$
\begin{aligned}
& \pi^{-1}(S)= \begin{cases}\left\{q^{i} \mid q^{i+1} \in S\right\} \cup\left\{q_{f}\right\} & \text { if } q_{f} \in S \text { or } \exists q^{1} \in S \\
\left\{q^{i} \mid q^{i+1} \in S\right\} & \text { else. }\end{cases} \\
& \pi^{i}(S)=\left\{q^{i} \mid \exists 1 \leq j \leq i \text { s.t. } q^{j} \in S\right\} \cup\left\{q_{f} \mid q_{f} \in S\right\}
\end{aligned}
$$

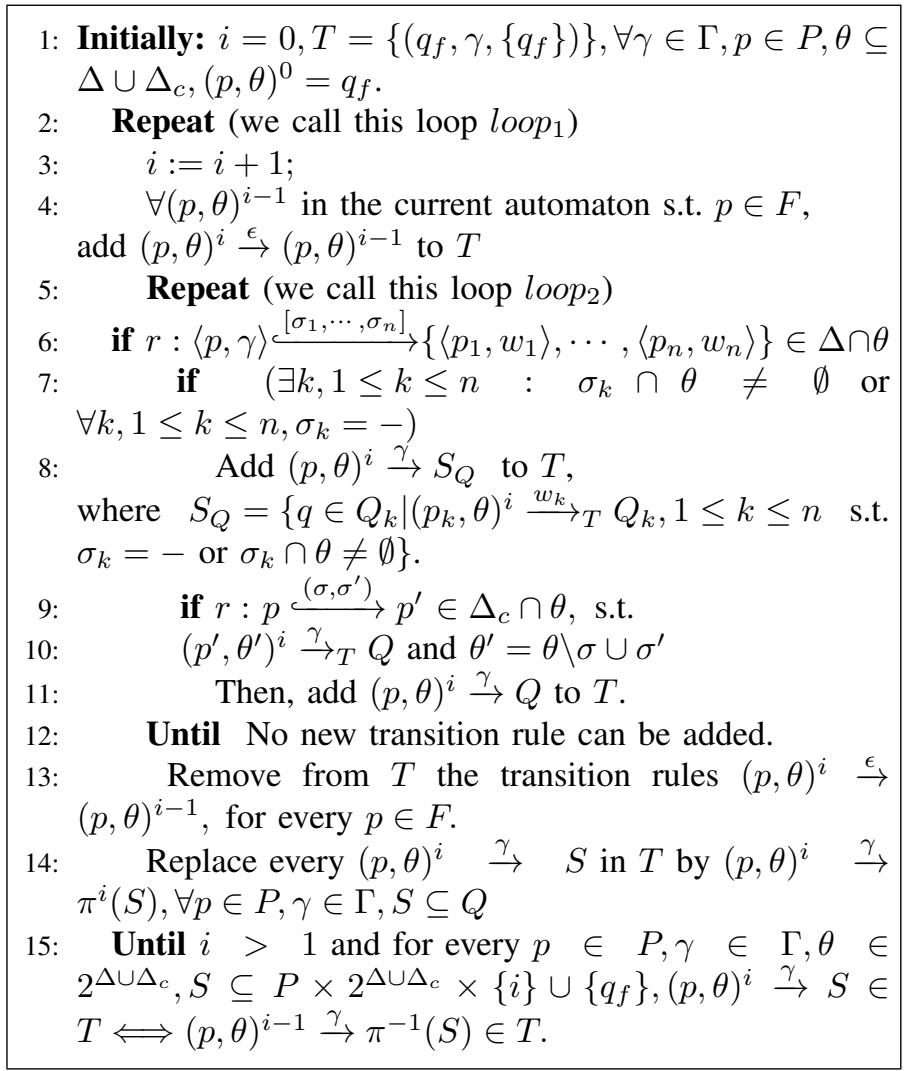

Algorithm 1: Computation of $Y_{\mathcal{B P}}$

Intuitively, at each step $i$, every state $(p, \theta)$ is represented by state $(p, \theta)^{i}$ in $\mathcal{A}_{i}$. For every $(p, \theta) \in I, \mathcal{A}_{i}$ recognizes a configuration $(\langle p, w\rangle, \theta)$ if $(p, \theta)^{i} \stackrel{\omega}{\rightarrow}_{T} q_{f} \cdot \mathcal{A}_{0}$ is the automaton obtained by Line 1. It accepts $X_{0}=P \times \Gamma^{*} \times 2^{\Delta \cup \Delta_{c}}$. At the beginning of each iteration, an $\epsilon$-transition in the form $(p, \theta)^{i} \stackrel{\epsilon}{\rightarrow} T(p, \theta)^{i-1}$ is added in Line 4 for every $(p, \theta) \in F \times 2^{\Delta \cup \Delta_{c}}$ in the current automaton. This allows to get $L\left(\mathcal{A}_{i-1}\right) \cap\left(F \times \Gamma^{*} \times 2^{\Delta \cup \Delta_{c}}\right)$. Lines 5-12 $\left(\right.$ loop $\left._{2}\right)$ is the saturation procedure that computes $\operatorname{Pre}_{\mathcal{B} \mathcal{P}}^{*}\left(L\left(\mathcal{A}_{i-1}\right) \cap(F \times\right.$ $\left.\left.\Gamma^{*} \times 2^{\Delta \cup \Delta_{c}}\right)\right)$. They ensure that if $\theta$ is a phase such that

$$
\langle p, \gamma\rangle \stackrel{\left[\sigma_{1}, \cdots, \sigma_{n}\right]}{\longrightarrow}\left\{\left\langle p_{1}, w_{1}\right\rangle, \cdots,\left\langle p_{n}, w_{n}\right\rangle\right\} \in \Delta \cap \theta
$$

, s.t. either $\exists k, 1 \leq k \leq n, \sigma_{k} \cap \theta \neq \emptyset$ or $\forall k, 1 \leq k \leq$ $n, \sigma_{k}=-$, and for every $k$ s.t. $\sigma_{k} \cap \theta \neq \emptyset$ or $\sigma_{k}=-$, $\left(\left\langle p_{k}, w_{k} w\right\rangle, \theta\right) \in L\left(\mathcal{A}_{i}\right)$ (i.e., $\left.\left(p_{k}, \theta\right)^{i} \stackrel{w_{k} w}{\longrightarrow} T q_{f}\right)$, then $(\langle p, \gamma w\rangle, \theta)$ should also be in $L\left(\mathcal{A}_{i}\right)$ (since it is a predecessor of $\left.\left\{\left(\left\langle p_{k}, w_{k} w\right\rangle, \theta\right), 1 \leq k \leq n\right\}\right)$. I.e., $T$ should contain the path $(p, \theta)^{i} \stackrel{\gamma w}{\longrightarrow}_{T} q_{f}$. This path is added thanks to Line 8 . Moreover, if $\theta$ is a phase such that $\langle p, \gamma\rangle \stackrel{\left(\sigma, \sigma^{\prime}\right)}{\longrightarrow} p^{\prime} \in \Delta_{c} \cap \theta$ and $\left(\left\langle p^{\prime}, \gamma w\right\rangle, \theta^{\prime}\right) \in L\left(\mathcal{A}_{i}\right)$ (i.e., $\left.\left(p^{\prime}, \theta^{\prime}\right)^{i} \stackrel{\gamma w}{\longrightarrow} q_{f}\right)$, where $\theta^{\prime}=\theta \backslash \sigma \cup \sigma^{\prime}$; then $(\langle p, \gamma w\rangle, \theta)$ should also be in $L\left(\mathcal{A}_{i}\right)$ (since it is a predecessor of $\left.\left(\left\langle p^{\prime}, \gamma w\right\rangle, \theta^{\prime}\right)\right)$. I.e., $T$ should contain the path $(p, \theta)^{i} \stackrel{\gamma w}{\longrightarrow}_{T} q_{f}$. This path is added thanks to Line 11 . Line 13 removes the $\epsilon$-transition added by Line 4 . This leads to $\operatorname{Pre}_{\mathcal{B} \mathcal{P}}^{+}\left(L\left(\mathcal{A}_{i-1}\right) \cap\left(F \times \Gamma^{*} \times 2^{\Delta \cup \Delta_{c}}\right)\right)$.

Let Algorithm A be Algorithm 1 without Line 14. Then, if Algorithm A terminates, it computes $Y_{\mathcal{B P}}$. However, if the sequence $X_{i}$ is strictly decreasing, Algorithm A never terminates. Lines 14-15 are then used to force termination. Indeed, thanks to the substitution of Line 14, at the end of step $i$, states of the form $(p, \theta)^{j}$, for $j<i$ become useless and can be removed. Line 15 checks then whether at step $i$, the transitions of $\mathcal{A}_{i}$ are "the same" than those of $\mathcal{A}_{i-1}$. If this is the case, the algorithm terminates. Termination of the algorithm then follows from the fact that step $i$ adds less transitions than step $i-1$. Intuitively, this is due to the fact that $L\left(\mathcal{A}_{i}\right) \subseteq L\left(\mathcal{A}_{i-1}\right)$, because step $i$ computes $\operatorname{Pr} e_{\mathcal{B} \mathcal{P}}^{+}\left(L\left(\mathcal{A}_{i-1}\right) \cap\left(F \times \Gamma^{*} \times 2^{\Delta \cup \Delta_{c}}\right)\right)$ and $\mathcal{A}_{0}$ accepts $P \times \Gamma^{*} \times 2^{\Delta \cup \Delta_{c}}$

Thus, we can show that (a detailed proof can be found in the full version [14]):

Theorem 3. Algorithm 1 always terminates and produces $Y_{\mathcal{B P}}$.

Thus, it follows from Theorems [, $\square$ and [] that:

Corollary 1. Let $\mathcal{P}$ be a SM-PDS, $\nu: P \rightarrow 2^{\text {At }}$ be a labelling function, and $\varphi$ be a CTL formula over At. Then, we can compute an EAMA $\mathcal{A}$ that characterizes the set of configurations $(\langle p, w\rangle, \theta)$ of $\mathcal{P}$ such that $(\langle p, w\rangle, \theta) \models_{\nu} \varphi$.

\section{EXPERIMENTS}

\section{A. Our algorithm vs. standard CTL on PDSs}

We implemented our algorithm in a tool and we compared its performance with the approach that consists in translating the SM-PDS to an equivalent standard PDS, and then applying the standard CTL model checking algorithm implemented in the PDS model-checker tool PuMoC [3] ]. All our experiments 


\begin{tabular}{|c|c|c|c|c|c|c|c|}
\hline \hline$|\Delta|+\left|\Delta_{c}\right|$ & $\begin{array}{c}\text { formula } \\
\text { size }\end{array}$ & $\begin{array}{c}\text { SM-PDS } \\
\text { time (s) }\end{array}$ & To PDS & PDS & Total Time & Result 1 & Result 2 \\
\hline $6+4$ & 5 & $\mathbf{0 . 3 6 s}$ & $0.21 \mathrm{~s}$ & $0.45 \mathrm{~s}$ & $0.66 \mathrm{~s}$ & $\mathrm{Y}$ & $\mathrm{Y}$ \\
$8+4$ & 12 & $\mathbf{2 . 8 8 s}$ & $0.35 \mathrm{~s}$ & $3.41 \mathrm{~s}$ & $3.76 \mathrm{~s}$ & $\mathrm{Y}$ & $\mathrm{Y}$ \\
$20+4$ & 15 & $\mathbf{3 . 8 4 s}$ & $0.62 \mathrm{~s}$ & $3.94 \mathrm{~s}$ & $4.56 \mathrm{~s}$ & $\mathrm{~N}$ & $\mathrm{~N}$ \\
$500+8$ & 6 & $\mathbf{2 0 . 5 1 s}$ & $17.02 \mathrm{~s}$ & $29.25 \mathrm{~s}$ & $46.27 \mathrm{~s}$ & $\mathrm{~N}$ & $\mathrm{~N}$ \\
$600+9$ & 8 & $\mathbf{2 3 . 3 4 s}$ & $295.24 \mathrm{~s}$ & $57.79 \mathrm{~s}$ & $353.03 \mathrm{~s}$ & $\mathrm{Y}$ & $\mathrm{Y}$ \\
$1000+10$ & 6 & $\mathbf{3 5 . 1 1 s}$ & $3251.02 \mathrm{~s}$ & $7127.41 \mathrm{~s}$ & $10378.43 \mathrm{~s}$ & $\mathrm{~N}$ & $\mathrm{~N}$ \\
$1100+10$ & 45 & $\mathbf{8 3 . 6 3 s}$ & $3251.02 \mathrm{~s}$ & - & - & $\mathrm{N}$ & - \\
$1500+8$ & 30 & $\mathbf{6 0 . 7 1 s}$ & $2182.78 \mathrm{~s}$ & $13821.34 \mathrm{~s}$ & $16004.12 \mathrm{~s}$ & $\mathrm{~N}$ & $\mathrm{~N}$ \\
$2000+10$ & 18 & $\mathbf{4 9 . 4 8 s}$ & $5529.30 \mathrm{~s}$ & - & - & $\mathrm{Y}$ & - \\
$2000+10$ & 36 & $\mathbf{6 1 . 1 3 s}$ & $5529.30 \mathrm{~s}$ & - & - & $\mathrm{N}$ & - \\
$2100+10$ & 15 & $\mathbf{6 0 . 7 4 s}$ & $5544.69 \mathrm{~s}$ & - & - & $\mathrm{Y}$ & - \\
$3800+10$ & 30 & $\mathbf{9 9 . 0 6 s}$ & $9295.24 \mathrm{~s}$ & - & - & $\mathrm{N}$ & - \\
$3850+10$ & 8 & $\mathbf{9 3 . 2 0 s}$ & $9308.01 \mathrm{~s}$ & - & - & $\mathrm{Y}$ & - \\
$3850+10$ & 30 & $\mathbf{1 1 5 . 5 2}$ & $9308.01 \mathrm{~s}$ & - & - & $\mathrm{N}$ & - \\
$4800+11$ & 10 & $\mathbf{1 4 2 . 1 3 s}$ & $42184.85 \mathrm{~s}$ & - & - & $\mathrm{Y}$ & - \\
$4800+11$ & 15 & $\mathbf{1 5 3 . 2 2}$ & $42184.85 \mathrm{~s}$ & - & - & $\mathrm{Y}$ & - \\
$5500+10$ & 20 & $\mathbf{1 9 6 . 4 6 s}$ & $45745.44 \mathrm{~s}$ & - & - & $\mathrm{Y}$ & - \\
$10180+16$ & 5 & $\mathbf{7 8 2 . 9 1 s}$ & $2134643.52 \mathrm{~s}$ & - & - & $\mathrm{N}$ & - \\
$10180+16$ & 8 & $\mathbf{1 0 4 1 . 8 7}$ & $2134643.52 \mathrm{~s}$ & - & - & $\mathrm{N}$ & - \\
$10180+16$ & 10 & $\mathbf{1 1 2 9 . 3 6 s}$ & $2134643.52 \mathrm{~s}$ & - & - & $\mathrm{Y}$ & - \\
\hline \hline
\end{tabular}

TABLE I: Our approach vs. standard CTL model checking for PDSs

were run on Ubuntu 16.04 with a $2.7 \mathrm{GHz} \mathrm{CPU}, 2 \mathrm{~GB}$ of memory. To perform this comparision, we randomly generate several SM-PDSs and CTL formulas. Our results (CPU Execution time) are shown in Table II. Column $|\Delta|+\left|\Delta_{c}\right|$ indicates the size of the transition rules. Column formula size shows the size of the CTL formula. Column SM-PDS is the cost of our direct algorithm. Column To PDS reports the cost it takes to get the equivalent PDS from the SM-PDS. Column PDS is the cost used to run standard CTL model checking for the equivalent PDS in PuMoC. Column Total Time is the whole cost it takes to translate the SM-PDS into a PDS, and then apply the PDS CTL model-checking algorithm of PuMoC [3]] $($ Total Time $=$ To PDS + PDS). Column Result 1 is the result of our approach and Result2 is the result of PuMoC [3]], where Y means yes the formula is satisfied and $\mathrm{N}$ means no, the formula is not satisfied. "-" means out of memory. It can be seen that our direct approach is much more efficient, and that it terminates in all the cases, whereas going through CTL model-checking of PDSs gets out of memory in most of the cases. Translating the SM-PDS to a standard PDS may take more than 24 days, whereas our direct algorithm takes only a few seconds. More results can be found in the full version [14].

\section{B. Malicious Behavior Detection on Self-Modifying Code}

1) Specifying malicious behaviors using CTL.: We applied our tool to detect several self-modifying malwares. Indeed, as shown in [32], several malicious behaviors can be described by CTL formulas. We give in what follows two examples of such malicious behaviors. More examples can be found in the full version [114].

Appending Viruses. An appending virus is a virus that inserts a copy of its code at the end of the target file. To achieve this, since the real OFFSET of the virus' variables depends on the size of the infected file, the virus has to first compute its real absolute address in the memory. To perform this, the virus has to call the sequence of instructions: $l_{1}$ : call $f ; l_{2}: \ldots ; f$ : pop eax; . The instruction call $f$ will push the return address $l_{2}$ onto the stack. Then, the pop instruction in $f$ will put the value of this address into the register eax. Thus, the virus can get its real absolute address from the register eax. This malicious behavior can be described by the following CTL formula:

$$
\phi_{a v}=\bigvee \mathbf{E F}(\text { call } \wedge \mathbf{E X} \text { (top-of-stack }=a) \wedge \mathbf{A G} \neg(\text { ret } \wedge
$$

$($ top-of-stack $=a))$ )

where the $\bigvee$ is taken over all possible return addresses $a$, and top-of-stack $=a$ is a predicate that indicates that the top of the stack is $a$. The subformula call $\wedge \mathbf{E X}$ (top-of-stack $=a$ ) means that there exists a procedure call having $a$ as return address. Indeed, when a procedure call is made, the program pushes its corresponding return address $a$ to the stack. Thus, at the next step, $a$ will be on the top of the stack. Therefore, the formula above expresses that there exists a procedure call having $a$ as return address, such that there is no ret instruction which will return to $a$.

Note that this formula uses predicates that indicate that the top of the stack is $a$. Our techniques work for this case as well: it suffices to encode the top of the stack in the control points of the SM-PDS. Our implementation works for this case as well and can handle appending viruses.

Spyware (Scanning the Disk). The aim of a spyware is to steal information from the host. To do this, it has to scan the disk of the host in order to find the interesting file that he wants to steal. If a file is found, it will run a payload to steal it, then continues searching the next file. If a directory is found, it will enter this path and continues scanning. This malicious behaviour is present e.g. in the notorious spyware Flame: It first calls the function FindFirstFileW to search the first object in the given path, then, it will check whether the function call succeeds or not. If the function call fails, it will call the function GetLastError. Otherwise it will call either the function FindFirstFileW again if it finds a directory or the 


\begin{tabular}{|c|c|c|c|c|c|c|c|c|c|c|c|}
\hline Example & Size & Result & cost & Example & Size & Result & cost & Example & Size & Result & cost \\
\hline calculation.exe & 9952 & No & $76.34 \mathrm{~s}$ & cisvc.exe & 4105 & No & $31.22 \mathrm{~s}$ & simple.exe & 52 & No & $3.17 \mathrm{~s}$ \\
\hline shutdown.exe & 2529 & No & $23.52 \mathrm{~s}$ & loop.exe & 529 & No & $11.78 \mathrm{~s}$ & cmd.exe & 1324 & No & $19.36 \mathrm{~s}$ \\
\hline notepad.exe & 10529 & No & $68.77 \mathrm{~s}$ & java.exe & 800 & No & $19.17 \mathrm{~s}$ & java.exe & 21324 & No & $122.07 \mathrm{~s}$ \\
\hline sort.exe & 8529 & No & $74.12 \mathrm{~s}$ & bibDesk.exe & 32800 & No & $243.79 \mathrm{~s}$ & interface.exe & 1005 & No & $18.25 \mathrm{~s}$ \\
\hline ipv4.exe & 968 & No & $24.43 \mathrm{~s}$ & TextWrangler.exe & 14675 & No & $65.09 \mathrm{~s}$ & sogou.exe & 45219 & No & $301.14 \mathrm{~s}$ \\
\hline game.exe & 34325 & No & $234.14 \mathrm{~s}$ & cycle.tex & 9014 & No & $75.44 \mathrm{~s}$ & calender.exe & 892 & No & $25.39 \mathrm{~s}$ \\
\hline Adson.1651 & 39 & Yes & $0.44 \mathrm{~s}$ & Adson. 1734 & 42 & Yes & $0.43 \mathrm{~s}$ & Alcaul.d & 40 & Yes & $0.48 \mathrm{~s}$ \\
\hline Adon.1703 & 37 & Yes & $0.39 \mathrm{~s}$ & Adon. 1559 & 37 & Ye s & $0.35 \mathrm{~s}$ & Alcaul.i & 48 & Yes & $0.44 \mathrm{~s}$ \\
\hline Alcaul.o & 33 & Yes & $0.29 \mathrm{~s}$ & Alcaul.d & 845 & Yes & $0.165 \mathrm{~s}$ & Alaul.c & 355 & Yes & $0.109 \mathrm{~s}$ \\
\hline Alcaul.j & 45 & Yes & $0.56 \mathrm{~s}$ & Alcaul.m & 23 & Yes & $0.19 \mathrm{~s}$ & Evol.a & 53 & Yes & $7.09 \mathrm{~s}$ \\
\hline Alcaul.e & 32 & Yes & $1.93 \mathrm{~s}$ & Alcaul.h & 34 & Yes & $3.95 \mathrm{~s}$ & Alcaul.g & 25 & Yes & $4.18 \mathrm{~s}$ \\
\hline Alcaul.b & 19 & Yes & $0.12 \mathrm{~s}$ & Alcaul.f & 23 & Yes & $1.99 \mathrm{~s}$ & Alcaul.k & 28 & Yes & $2.31 \mathrm{~s}$ \\
\hline Alcaul.1 & 27 & Yes & $0.95 \mathrm{~s}$ & Klinge & 45 & Yes & $64.15 \mathrm{~s}$ & Akez.Win32.5 & 490 & Yes & $53.18 \mathrm{~s}$ \\
\hline Netsky.a & 45 & Yes & $19.12 \mathrm{~s}$ & Mydoom.c & 155 & Yes & $4.14 \mathrm{~s}$ & MyDoom-N & 16980 & Yes & $343.93 \mathrm{~s}$ \\
\hline Netsky.x & 55 & Yes & $21.85 \mathrm{~s}$ & Netsky.y & 68 & Yes & $29.06 \mathrm{~s}$ & Netsky.z & 115 & Yes & $43.37 \mathrm{~s}$ \\
\hline Netsky.gen & 5508 & Yes & $59.24 \mathrm{~s}$ & Netsky.p & 6015 & Yes & $76.32 \mathrm{~s}$ & Netsky.m & 6805 & Yes & $73.77 \mathrm{~s}$ \\
\hline Netsky.r & 230 & Yes & $8.83 \mathrm{~s}$ & Netsky.k & 6115 & Yes & $79.79 \mathrm{~s}$ & Netsky.e & 6245 & Yes & $79.44 \mathrm{~s}$ \\
\hline Mydoom.y & 26902 & Yes & $452.77 \mathrm{~s}$ & Mydoom.j & 22355 & Yes & $211.93 \mathrm{~s}$ & klez-N & 6281 & Yes & $63.07 \mathrm{~s}$ \\
\hline klez.c & 30 & Yes & $2.79 \mathrm{~s}$ & Mydoom.v & 5965 & Yes & $283.11 \mathrm{~s}$ & Netsky.b & 45 & Yes & $29.51 \mathrm{~s}$ \\
\hline LdPinch.dd & 8230 & Yes & $48.17 \mathrm{~s}$ & LdPinch.gw & 2050 & Yes & $39.92 \mathrm{~s}$ & LdPinch.dh & 3744 & Yes & $41.01 \mathrm{~s}$ \\
\hline LdPinch.c & 7363 & Yes & $52.46 \mathrm{~s}$ & LdPinch.ck & 8228 & Yes & $49.77 \mathrm{~s}$ & LdPinch.gen & 7245 & Yes & $62.19 \mathrm{~s}$ \\
\hline LdPinch.ld & 6631 & Yes & $29.70 \mathrm{~s}$ & LdPinch.er & 3910 & Yes & $32.73 \mathrm{~s}$ & LdPinch.cf & 4366 & Yes & $27.26 \mathrm{~s}$ \\
\hline Muma.c & 15915 & Yes & $114.47 \mathrm{~s}$ & LdPinch.au & 8245 & Yes & $92.24 \mathrm{~s}$ & LdPinch.fi & 3138 & Yes & $74.39 \mathrm{~s}$ \\
\hline Gismor & 1140 & Yes & $23.56 \mathrm{~s}$ & Botter.a & 5030 & Yes & $71.42 \mathrm{~s}$ & Navidad.a & 3750 & Yes & $46.59 \mathrm{~s}$ \\
\hline Bagle.ab & 5690 & Yes & $89.42 \mathrm{~s}$ & Bagle.ef & 995 & Yes & $54.11 \mathrm{~s}$ & Bagle.eg & 380 & Yes & $25.49 \mathrm{~s}$ \\
\hline Atak.f & 2005 & Yes & $11.35 \mathrm{~s}$ & Atak.g & 2498 & Yes & $16.69 \mathrm{~s}$ & Atak.l & 1914 & Yes & $10.37 \mathrm{~s}$ \\
\hline Newapt.C & 11730 & Yes & $924.92 \mathrm{~s}$ & Krynos.b & 18370 & Yes & $893.45 \mathrm{~s}$ & Jeans.a & 6490 & Yes & $188.36 \mathrm{~s}$ \\
\hline Bagle.m & 5111 & Yes & $39.92 \mathrm{~s}$ & Bagle.k & 35 & Yes & $1.92 \mathrm{~s}$ & Bagle.t & 3345 & Yes & $45.64 \mathrm{~s}$ \\
\hline LdPinch.aaz & 4145 & Yes & $41.05 \mathrm{~s}$ & LdPinch.c0 & 8230 & Yes & $65.17 \mathrm{~s}$ & LdPinch.ee & 6501 & Yes & $71.30 \mathrm{~s}$ \\
\hline LdPinch.v & 7235 & Yes & $51.69 \mathrm{~s}$ & LdPinch.fk & 4906 & Yes & $47.11 \mathrm{~s}$ & LdPinch.awp & 195 & Yes & $17.97 \mathrm{~s}$ \\
\hline LdPinch.bb & 8145 & Yes & $63.13 \mathrm{~s}$ & LdPinch.br & 3645 & Yes & $33.52 \mathrm{~s}$ & LdPinch.hb & 1645 & Yes & $21.08 \mathrm{~s}$ \\
\hline LdPinch.by & 970 & Yes & $42.92 \mathrm{~s}$ & Generic.2026199 & 433 & Yes & $32.83 \mathrm{~s}$ & LdPinch.arr & 1250 & Yes & $49.84 \mathrm{~s}$ \\
\hline Mydoom.M & 5965 & Yes & $75.19 \mathrm{~s}$ & MyDoom.54464 & 5935 & Yes & $45.78 \mathrm{~s}$ & Mydoom.e & 138 & Yes & $46.53 \mathrm{~s}$ \\
\hline Mydoom.ACQ & 19210 & Yes & $439.57 \mathrm{~s}$ & Mydoom.ba & 19423 & Yes & $238.77 \mathrm{~s}$ & Mydoom.ftde & 19495 & Yes & $339.29 \mathrm{~s}$ \\
\hline Sramota.avf & 240 & Yes & $11.01 \mathrm{~s}$ & Mydoom & 238 & Yes & $2.01 \mathrm{~s}$ & Mydoom.288 & 248 & Yes & $3.12 \mathrm{~s}$ \\
\hline Mydoom.R & 230 & Yes & $30.22 \mathrm{~s}$ & Mydoom.dlnpqi & 235 & Yes & $1.99 \mathrm{~s}$ & Mydoom.o & 235 & Yes & $2.01 \mathrm{~s}$ \\
\hline klez.e & 27 & Yes & $3.94 \mathrm{~s}$ & Magistr.b & 4670 & Yes & $231.97 \mathrm{~s}$ & Magistr.a.poly & 36989 & Yes & $469.63 \mathrm{~s}$ \\
\hline Kelino.g & 470 & Yes & $22.08 \mathrm{~s}$ & Plage.b & 395 & Yes & $1.96 \mathrm{~s}$ & Urbe.a & 123 & Yes & $9.17 \mathrm{~s}$ \\
\hline Kelino.1 & 495 & Yes & $21.01 \mathrm{~s}$ & Kipis.t & 20378 & Yes & $121.11 \mathrm{~s}$ & klez.d & 31 & Yes & $0.95 \mathrm{~s}$ \\
\hline Netsky.d & 45 & Yes & $1.87 \mathrm{~s}$ & Ardurk.d & 1913 & Yes & $12.08 \mathrm{~s}$ & klez.f & 27 & Yes & $0.73 \mathrm{~s}$ \\
\hline Repah.b & 221 & Yes & $12.76 \mathrm{~s}$ & Gibe.b & 5358 & Yes & $37.01 \mathrm{~s}$ & Magistr.b & 4670 & Yes & $43.59 \mathrm{~s}$ \\
\hline NGVCK1 & 329 & Yes & $2.33 \mathrm{~s}$ & NGVCK2 & 455 & Yes & $2.89 \mathrm{~s}$ & NGVCK3 & 2300 & Yes & $111.20 \mathrm{~s}$ \\
\hline NGVCK4 & 550 & Yes & $9.19 \mathrm{~s}$ & NGVCK5 & 1555 & Yes & $10.25 \mathrm{~s}$ & NGVCK6 & 1698 & Yes & $21.07 \mathrm{~s}$ \\
\hline NGVCK7 & 6902 & Yes & $14.24 \mathrm{~s}$ & NGVCK8 & 2355 & Yes & $54.76 \mathrm{~s}$ & NGVCK9 & 281 & Yes & $12.31 \mathrm{~s}$ \\
\hline NGVCK10 & 2980 & Yes & $23.51 \mathrm{~s}$ & NGVCK11 & 5965 & Yes & $51.92 \mathrm{~s}$ & NGVCK12 & 4529 & Yes & $36.14 \mathrm{~s}$ \\
\hline NGVCK13 & 2210 & Yes & $18.12 \mathrm{~s}$ & NGVCK14 & 5358 & Yes & $120.04 \mathrm{~s}$ & NGVCK15 & 970 & Yes & $42.12 \mathrm{~s}$ \\
\hline NGVCK16 & 658 & Yes & $6.59 \mathrm{~s}$ & NGVCK17 & 913 & Yes & $2.03 \mathrm{~s}$ & NGVCK18 & 90 & Yes & $0.89 \mathrm{~s}$ \\
\hline NGVCK19 & 1295 & Yes & $16.58 \mathrm{~s}$ & NGVCK20 & 4378 & Yes & $32.41 \mathrm{~s}$ & NGVCK21 & 31 & Yes & $0.77 \mathrm{~s}$ \\
\hline NGVCK 22 & 370 & Yes & $2.08 \mathrm{~s}$ & NGVCK23 & 3955 & Yes & $36.74 \mathrm{~s}$ & NGVCK24 & 6924 & Yes & $92.03 \mathrm{~s}$ \\
\hline
\end{tabular}

TABLE II: Partial Experimental Results

\begin{tabular}{|c|c|c|c|c|c|c|c|c|c|c|}
\hline our tool & McAfee & Norman & BitDefender & Kinsoft & Avira & eScan & Kaspersky & Qihoo360 & Avast & Symantec \\
\hline $\mathbf{1 0 0 \%}$ & $38.0 \%$ & $33.3 \%$ & $48.5 \%$ & $31.4 \%$ & $11.6 \%$ & $21.9 \%$ & $70.4 \%$ & $1.4 \%$ & $2.3 \%$ & $57.1 \%$ \\
\hline
\end{tabular}

TABLE III: Our tool v.s. well known anti-viruses

function FindNextFileW to search for the next object. We can specify this behavior in CTL as follows:

$$
\begin{aligned}
\phi_{\text {spy }}=\mathbf{E F} & (\text { call FindFirstFileW } \wedge \mathbf{A F}(\text { call GetLastError } \\
& \vee \text { call FindFirstFileW } \vee \text { call FindNextFileW }))
\end{aligned}
$$

This formula states that there exists a path where the function FindFirstFileW is called, then, in all the future paths, the program either calls GetLastError (if FindFirstFileW failed) or calls FindFirstFileW (if a directory is found) or calls FindNextFileW (to search for the next file). Scanning a disk can be a behavior of a benign program. To avoid false alarms, we can combine this CTL formula with other formulas describing other malicious behaviors expressing the payload (such as sending a file) to determine whether the binary code is a malware or not. Note that, the formula is branching time and cannot be described as a LTL formula.

2) Applying our tool for malware detection.: We applied our tool to detect several malwares. We use Jakstab [29] as disassembler. We consider 400 email-worms, 30 worms and 100 viruses from VX heaven[33] and 260 new malwares generated by NGVCK, one of the best malware generators. We also 
choose 19 benign samples from Windows XP system (win32). We consider self-modifying versions of these programs. In these versions, the malicious behaviors are unreachable if the semantics of the self-modifying instructions are not taken into account, i.e., if the self-modifying instructions are considered as "standard" instructions that do not modify the code, then the malicious behaviors cannot be reached. First, we abstract away the semantics of the self-modifying instructions and model such programs as standard PDSs as described in [32], and perform CTL model-checking for PDSs to determine whether the programs contain any malicious behavior. In this case, none of the programs was declared as malicious. Then, we use SM-PDSs to model these programs, thus, taking selfmodifying instructions into consideration. Then, we check whether these SM-PDSs satisfy any malicious CTL formula in our database (CTL formulas described above and in the full version). If yes, the program is declared as malicious. If not, it is declared as benign. In our experiments (we have 790 malwares), our tool was able to detect all these programs as malicious (whereas when we model these programs using standard PDSs and abstract away self-modifying instructions, none of these programs was detected as malicious). Our tool was also able to determine that benign programs are benign. We report in Table III some of the results we obtained. More results can be found in the full version. Column Size gives the number of control locations, Column Result shows the result of our algorithm: $\mathrm{Y}$ means malicious and $\mathrm{N}$ means benign; and Column cost gives the cost in seconds. You can see that our CTL model checking approach allows to detect all the malicious programs in a few seconds.

3) Comparision with well-known antiviruses.: We compare our tool against well-known and widely used antiviruses such as McAfee, Norman, BitDefender, Kinsoft, Avira, eScan, Kaspersky, Qihoo-360, Avast, and Symantec. To have a fair comparision, we need to consider unknown malwares. Thus, we generated 105 malwares that include self-modifying codes. We use the sophisticated malware generator NGVCK available at VX Heavens [33] to obtain malicious codes and we obfuscate them using self-modifying instructions. Our tool was able to detect all these programs as malicious, whereas none of the well-known antiviruses was able to detect all these malwares. Table III shows the detection rates of our tool v.s. the wellknown anti-viruses.

\section{REFERENCES}

[1] J. Bergeron, M. Debbabi, J. Desharnais, M. M. Erhioui, Y. Lavoie, N. Tawbi et al., "Static detection of malicious code in executable programs," Int. J. of Req. Eng, vol. 2001, no. 184-189, p. 79, 2001.

[2] G. Balakrishnan, T. W. Reps, N. Kidd, A. Lal, J. Lim, D. Melski, R. Gruian, S. H. Yong, C. Chen, and T. Teitelbaum, "Model checking x86 executables with codesurfer/x86 and WPDS++," in $C A V, 2005$, pp. $158-163$.

[3] P. K. Singh and A. Lakhotia, "Static verification of worm and virus behavior in binary executables using model checking," in $I A W, 2003$, pp. 298-300.

[4] M. Christodorescu and S. Jha, "Static analysis of executables to detect malicious patterns," University of Wisconsin-Madison Department of Computer Sciences, Tech. Rep., 2003.

[5] J. Kinder, S. Katzenbeisser, C. Schallhart, and H. Veith, "Detecting malicious code by model checking," in DIMVA, 2005, pp. 174-187.
[6] F. Song and T. Touili, "Pushdown model checking for malware detection," STTT, vol. 16, no. 2, pp. 147-173, 2014.

[7] M. Christodorescu, S. Jha, S. A. Seshia, D. Song, and R. E. Bryant, "Semantics-aware malware detection," in S\&P, 2005, pp. 32-46.

[8] G. Bonfante, H. Godfroy, and J.-Y. Marion, "A construction of a selfmodifiying language with a formal correction proof," in MALWARE. IEEE, 2017, pp. 99-106.

[9] E. R. Team, "Self-modifying code unpacking tool using dynamorio," https://github.com/BreakıngMalware/Selne.

[10] Karl, "Automated unpacking: A behaviour based approach," https:// github.com/malwaremusings/unpacker.

[11] T. Touili and X. Ye, "Reachability analysis of self modifying code," in ICECCS. IEEE, 2017, pp. 120-127.

[12] A. Lakhotia, E. U. Kumar, and M. Venable, "A method for detecting obfuscated calls in malicious binaries," IEEE Trans. Software Eng., vol. 31, no. 11, pp. 955-968, 2005. [Online]. Available: https://do1.org/I0.1109/TSE.2005.120

[13] F. Song and T. Touili, "Efficient ctl model-checking for pushdown systems," Theoretical Computer Science, vol. 549, pp. 127-145, 2014.

[14] T.Touili and X.Ye, "Ctl model checking of self modifying code," https: //lipn.univ-paris 13.tr/ xin/ctl_full.pdt.

[15] F. Song and T. Touili, "Efficient malware detection using modelchecking," in $F M, 2012$, pp. 418-433.

[16] P. Beaucamps, I. Gnaedig, and J.-Y. Marion, "Behavior abstraction in malware analysis," in International Conference on Runtime Verification. Springer, 2010, pp. 168-182.

[17] F. Song and T. Touili, "Ltl model-checking for malware detection," in TACAS. Springer, 2013, pp. 416-431.

[18] H. Nguyen and T. Touili, "CARET model checking for malware detection," in SPIN 2017, H. Erdogmus and K. Havelund, Eds. ACM, 2017, pp. 152-161. [Online]. Available: https://do1.org/10.1145/ 3(1)92282.3(1)923()

[19] G. Bonfante, J.-Y. Marion, and D. Reynaud-Plantey, "A computability perspective on self-modifying programs," in SEFM, 2009, pp. 231-239.

[20] H. Cai, Z. Shao, and A. Vaynberg, "Certified self-modifying code," ACM SIGPLAN Notices, vol. 42, no. 6, pp. 66-77, 2007.

[21] S. K. D. K. P. Coogan and G. M. Townsend, "On the semantics of self-unpacking malware code,' Citeseer, Tech. Rep., 2008.

[22] B. Anckaert, M. Madou, and K. De Bosschere, "A model for selfmodifying code," in $I H, 2006$, pp. 232-248.

[23] S. Blazy, V. Laporte, and D. Pichardie, "Verified abstract interpretation techniques for disassembling low-level self-modifying code," Journal of Automated Reasoning, vol. 56, no. 3, pp. 283-308, 2016.

[24] K. A. Roundy and B. P. Miller, "Hybrid analysis and control of malware," in International Workshop on Recent Advances in Intrusion Detection. Springer, 2010, pp. 317-338.

[25] K. Coogan, S. Debray, T. Kaochar, and G. Townsend, "Automatic static unpacking of malware binaries," in WCRE, 2009, pp. 167-176.

[26] M. G. Kang, P. Poosankam, and H. Yin, "Renovo: A hidden code extractor for packed executables," in WORM. ACM, 2007, pp. 4653.

[27] P. Royal, M. Halpin, D. Dagon, R. Edmonds, and W. Lee, "Polyunpack: Automating the hidden-code extraction of unpack-executing malware," in $A C S A C, 2006$, pp. 289-300.

[28] I. Walukiewicz, "Model checking ctl properties of pushdown systems," in International Conference on Foundations of Software Technology and Theoretical Computer Science. Springer, 2000, pp. 127-138.

[29] V. H. Kinder.J., "Jakstab: A static analysis platform for binaries," in $C A V$. Springer, 2008, pp. 423-427.

[30] A. Bouajjani, J. Esparza, and O. Maler, "Reachability Analysis of Pushdown Automata: Application to Model Checking," in CONCUR, 1997.

[31] F. Song and T. Touili, "Pumoc: a ctl model-checker for sequential programs," in ASE, 2012, pp. 346-349.

[32] — " "Efficient malware detection using model-checking," in International Symposium on Formal Methods. Springer, 2012, pp. 418-433.

[33] V.Heaven, "V.heavens," http://vxer.org/lib/. 